\title{
Granular suspension avalanches. I. Macro-viscous behavior
}

\author{
Christophe Ancey, Nicolas Andreini, and Gaël Epely-Chauvin ${ }^{\text {a) }}$ \\ École Polytechnique Fédérale de Lausanne, Écublens, 1015 Lausanne, Switzerland
}

(Received 7 August 2012; accepted 25 January 2013; published online 6 March 2013)

\begin{abstract}
We experimentally studied the flow behavior of a fixed volume of granular suspension, initially contained in a reservoir and released down an inclined flume. Here "granular suspension" refers to a suspension of non-Brownian particles in a viscous fluid. Depending on the solids fraction, density mismatch, and particle size distribution, a wealth of behaviors can be observed. Here we report and interpret results obtained with granular suspensions, which consisted of neutrally buoyant particles with a solids fraction $(\phi=0.575-0.595)$ close to the maximum random packing fraction (estimated at $\phi_{m}=0.625$ ). The particles had the same refractive index as the fluid, which made it possible to measure the velocity profiles inside the moving bulk and far from the sidewalls. Additional information such as the front position and the flow depth was also recorded. Three regimes were observed. At early times, the flow features were reminiscent of homogeneous Newtonian fluids (e.g., the same dependence of the front position on time). At later times, the free surface became more and more bumpy as fractures developed within the bulk. This fracture process ultimately gave rise to a stick-slip regime, in which the suspension moved intermittently. In this paper, we focus on the first regime referred to as the macroviscous regime. Although the bulk flow properties looked like those of Newtonian fluids, the internal dynamics were much richer. @ 2013 American Institute of Physics. [http://dx.doi.org/10.1063/1.4793719]
\end{abstract}

\section{INTRODUCTION}

Granular suspensions are defined as concentrated suspensions of noncolloidal particles within viscous fluids. ${ }^{1,2}$ We bear in mind here that in such a granular suspension, there are many contacts between particles, specifically continuous physical interactions arising from lubricated or direct solid/frictional contacts. This leads to percolating networks of particles, which form, evolve, and break continuously throughout the suspension., ${ }^{2,3}$ The presence of a particle network demonstrates that granular suspensions exhibit long-range spatial ordering of their microstructure (i.e., the particle arrangement), which creates a coupling between the local behavior and bulk motion. This gives rise to a number of effects present in dry granular materials such as dilatancy, ${ }^{4,5}$ shear banding, ${ }^{6}$ particle jamming, ${ }^{7,8}$ and frictional behavior. ${ }^{9,10}$ At the same time, the grains are saturated with viscous fluids and inherit some of the features of concentrated suspensions such as shear thickening, particle migration, and normal stress effects. ${ }^{11,12}$

Since granular suspensions exhibit properties typical of both granular materials and viscous non-Newtonian fluids, it is natural to wonder whether these distinctive properties are present at the same time or whether, on the contrary, they are mutually exclusive, i.e., they are intrinsic to either viscous or frictional regimes. In the latter case, another related question is the delineation of these regimes. While this issue has attracted growing attention over the last decade, no conclusive answer has been found. For instance, for non-buoyant particles, Ancey ${ }^{13}$ and Ancey and Coussot ${ }^{10}$ used a Couette cell to investigate the rheometric behavior of concentrated suspensions

\footnotetext{
a) Deceased.
} 
composed of glass beads in various interstitial fluids. By varying the flow depth and using dimensional analysis, these authors provided evidence that at low shear velocities, the behavior was clearly frictional (with a typical Coulomb response of shear stress), while at high shear velocities, the behavior resembled that of a viscous Newtonian material (with a linear dependence of shear stress on shear rate). Using an annular shear cell, Prasad and Kytömaa ${ }^{9}$ also observed a transition from a frictional to a viscous regime when shearing the sample at constant solids fraction. In contrast with measurements taken with a Couette cell, the shear stress was a decreasing function of the shear rate in the frictional regime, which led to instabilities (wide fluctuations were observed).

A few authors conducted dam-break or fully developed flow experiments to gain insight into the rheological behavior of concentrated suspensions. Nsom ${ }^{14}$ ran dam-break experiments with neutrally buoyant particle suspensions (acrylic plastic beads immersed in glucose solutions) with solid concentration as high as 0.60 . He found that for solid concentrations lower than 0.52 , the flow features (flow depth profile and position of the front with time) looked like those of a viscous flow, but for higher concentrations (typically 0.60 ), he observed that the flow came to a halt, a phenomenon typical of plastic behavior. Timberlake and Morris ${ }^{15}$ measured the velocity and concentration profiles in thin films of concentrated suspensions flowing down an inclined plane for various inclinations and concentrations. The data were compared with the predictions from a particle-migration model. They observed that the free surface became more deformed with increasing concentration or inclination. Bonnoit et al. ${ }^{16}$ used $40 \mu \mathrm{m}$ polystyrene beads suspended in silicone oils in the 0.35-0.61 solids fraction range. By using the steady-state equation that relates the bulk viscosity to the surface velocity and flow depth, they were able to measure the bulk viscosity, which was consistent with Zarraga's empirical equation. Non-buoyant particle suspensions were also used in flume experiments. Ward et al. ${ }^{17}$ carried out experiments with solids fractions ranging from 0.35 to 0.55 . Various input parameters (particle density and size, fluid viscosity, initial volume, plane inclination) were varied. They showed that the scaling of the front position with time was properly described using lubrication theory. Zhou et al. ${ }^{18}$ observed that for moderately concentrated suspensions and gentle slopes, the suspension remained well mixed and flowed like a viscous fluid, but for highly concentrated suspensions and steep slopes, particles moved faster than the interstitial fluid and accumulated in a ridge just behind the contact line.

A particular difficulty in the rheological study of coarse particle suspensions is related to the very nature of the data used for analyzing the rheological properties. In most cases, these data have been obtained from macroscopic measurements (e.g., using rheometers), assuming that the bulk behaves as a homogeneous fluid, however there is accumulating evidence that this procedure leads to miscalculations of the bulk behavior. ${ }^{19,20}$ The current trend is to infer rheological properties by local measurements. Flow visualization has made it possible to derive the rheological properties directly by measuring the local velocity and particle concentrations. For instance, using magnetic resonance imaging, Ovarlez, Bertrand, and Rodts ${ }^{20}$ measured the velocity and concentration profiles inside a wide-gap coaxial-cylinder rheometer. They found that, due to particle migration, the local rheology was markedly different from the bulk rheological properties derived from viscometry theory.

In this article, we report the macro-viscous behavior observed for perfectly density-matched suspensions flowing down an inclined flume. We start with a description of the experimental procedure (see Sec. II). A fundamental part of the experiments was the optimal control of the suspension properties, especially the refractive index of solid and fluid phases, and their density difference, a point that is crucial for the proper interpretation of the results. ${ }^{21,22}$ In Sec. III, we present the results by focusing not only on the bulk flow properties (flow-depth profile, front position with time) but also on the velocity profiles inside the flow. We failed to measure the particle concentration to a sufficient accuracy. Indeed, in time-dependent flows of highly concentrated suspensions, optical methods lead to uncertainties that are not compatible with the precision desired. We end the paper with concluding remarks. To keep the body of this paper as coherent and concise as possible, we have moved the theoretical material to the Appendix. This appendix aids understanding of the experimental results. 

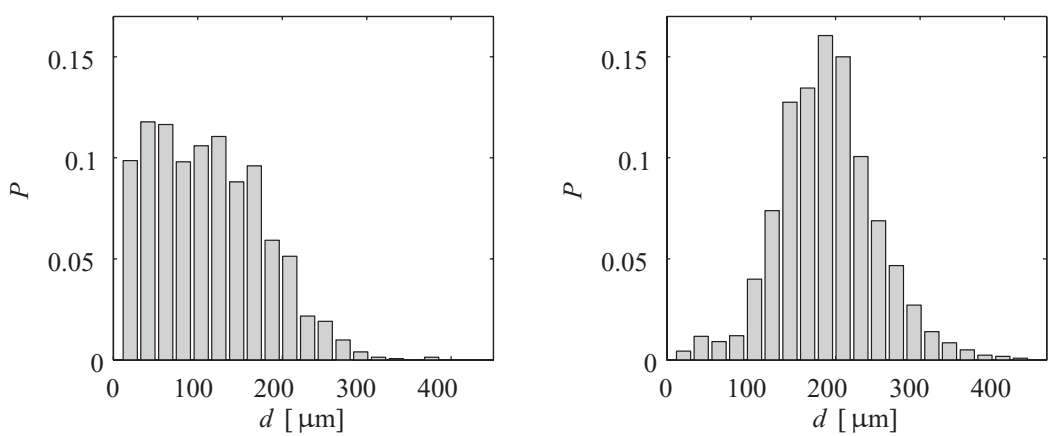

FIG. 1. Grain size distributions of PMMA particles used in our experiments. Histograms were obtained by measuring particles' diameters with a microscope (Olympus BX60). (Left) Raw grain size distribution (sample size: 1520 particles). (Right) Grain size distribution after sieving (sample size: 3427 particles).

\section{EXPERIMENTAL FACILITY AND PROCEDURE}

\section{A. Suspension composition}

All experiments were run with highly concentrated suspensions of poly(methyl methacrylate) (PMMA) particles in a Newtonian fluid. We explored solids fractions in the $0.30-0.61$ range, ${ }^{23}$ but here we present only the data corresponding to the $0.575-0.595$ range. Considerable attention was paid to obtaining transparent density-matched suspensions as even slight density contrasts in highly concentrated suspensions can lead to experimental artifacts. ${ }^{21}$ The density mismatch between the fluid and solid phases was zero to within $5 \times 10^{-4} \mathrm{~g} \mathrm{~cm}^{-3}$ at $20^{\circ} \mathrm{C}$, a remarkably low value. The particles and fluids had the same refractive index to within $10^{-4}$.

Two size distributions were used: the first distribution, hereafter referred to as unsieved, consisted of the raw material provided by Altuglass. The mean particle diameter was $110 \mu \mathrm{m}$ (standard deviation $65 \mu \mathrm{m})$. The second distribution was obtained by sieving the raw material using a sieving machine (Retsch AS200 Control) with a $180 \mu \mathrm{m}$ sealed sieving stack. The mean particle diameter was $190 \mu \mathrm{m}$ (standard deviation $60 \mu \mathrm{m}$ ). Figure 1 shows that for the unsieved distribution, the grain size distribution was rather uniform, with most particles ranging from $15 \mu \mathrm{m}$ to $200 \mu \mathrm{m}$, while the second distribution was bell-shaped and much richer in coarse particles. Oedometric tests showed that for loose samples, the permeability of the unsieved material was $k=4 \times 10^{-12} \mathrm{~m}^{2}$ (a much lower value than that given by the Kozeny-Carman equation for monosized particles). The random close packing $\phi_{R C P}$ was measured by pouring a mass $M_{p}$ of dry beads into a calibrated round-bottom flask of volume $V_{c}$, and vibrating the cylinder until steady compaction occurred. We found $\phi_{R C P}$ $=0.655 \pm 0.02$. We were not able to detect significant influence of the particle distribution on $\phi_{R C P}$.

The carrier fluid was a mixture of fluids called trimix of viscosity $0.124 \pm 0.003 \mathrm{~Pa}$ s at $20^{\circ} \mathrm{C}$, composed of 50\% Triton X100, $28 \%$ 1,6-Dibromohexane, and 22\% UCON oil (75-H450 oil from Dow Chemicals). The final bulk density was $\rho=1.184 \pm 0.0005 \mathrm{~g} / \mathrm{cm}^{3}$, the refractive index at 532 $\mathrm{nm}$ and $20^{\circ} \mathrm{C}$ was $1.48850 \pm 0.00025$. The surface tension was measured using the pendant drop method: ${ }^{24}$ we found $\gamma=33 \pm 5 \mathrm{mN} \mathrm{m}^{-1}$.

\section{B. Flume}

Experiments were conducted in a PMMA-bottomed flume with aluminum sidewalls. Figure 2 shows a sketch of the facility. An optical glass pane, located $50 \mathrm{~cm}$ from the flume outlet, was inserted to observe the flows from the side. The flume was $3.5 \mathrm{~m}$ long and $10 \mathrm{~cm}$ wide. It could be inclined from $0^{\circ}$ to $35^{\circ}$, but all the experimental data reported here were obtained with a slope $\theta=25^{\circ}$. Its position was accurately controlled using a digital inclinometer with a precision of $0.1^{\circ}$. The upper part of flume was equipped with a sluice gate mounted on a pneumatic jack and was used as a reservoir. The jack was quickly raised by injecting air pressurized at $7 \mathrm{MPa}$, which made it possible to lift the gate within $0.5 \mathrm{~s}$. 


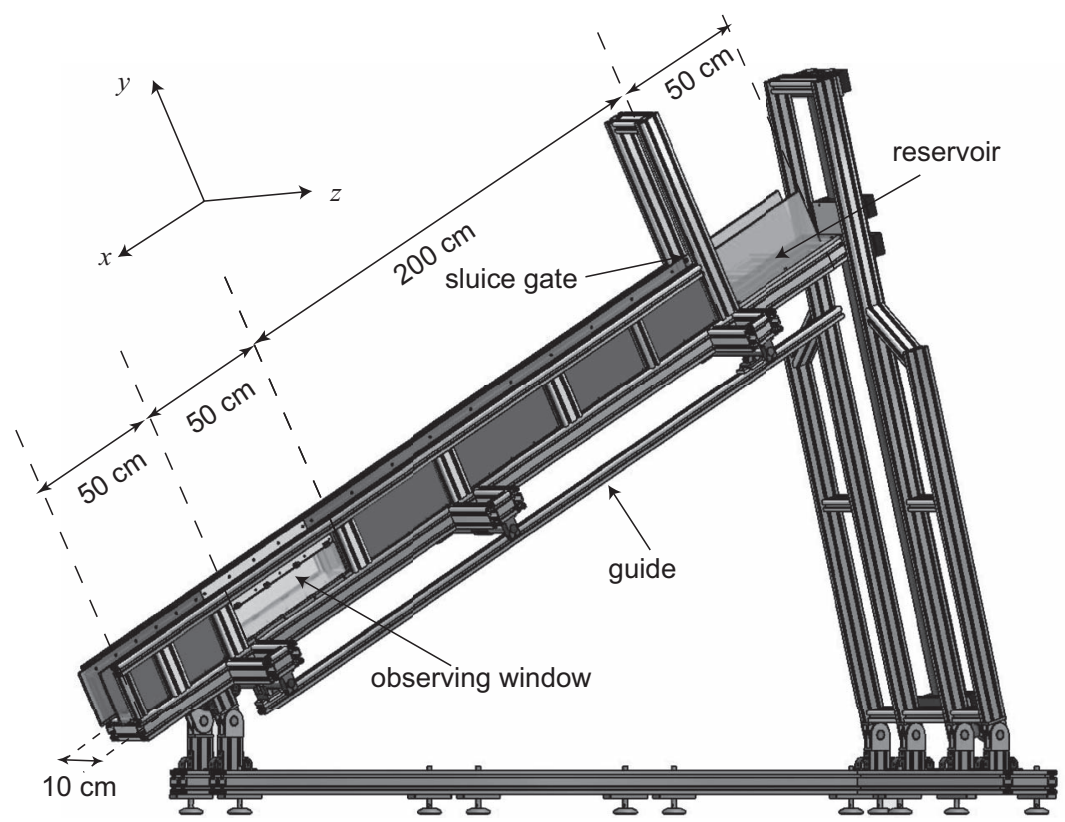

FIG. 2. Flume used for the experiments.

\section{Measurement systems}

We took the following measurements:

- Flow depth profiles in the downstream and cross-stream directions (normal to the sidewall).

- Velocity profiles in the direction normal to the flume bottom.

- Position of the front as a function of time.

Given how difficult it was to operate all the devices simultaneously, some of the measurements (e.g., pore fluid pressure and cross-stream depth profile) were taken only for some runs. We used two types of laser:

- A dual-head, diode-pumped, Q-switched Nd:YLF Laser (Litron LDY 303). The laser had two optical cavities emitting a $527 \mathrm{~nm}$ beam (green), with energy up to $20 \mathrm{~mJ}$ per pulse at $1 \mathrm{kHz}$. We also used lenses and a splitter to split the main laser beam into two beams and focus it.

- Laser diodes emitting $670 \mathrm{~nm}$ beams (red) to measure the free-surface deformations.

Flow depths and velocities were measured using high-speed cameras and particle image velocimetry (PIV) techniques. Figure 3 explains how we measured the velocity profiles from below using the Scheimpflug principle (for the filmed plane to be completely in focus). It was also possible to take images from the side for uniform flows, but it was much more constraining from the optical standpoint for nonuniform flows (in particular, the shorter optical path length was obtained with the camera placed below the flume bottom). For PIV measurements, we used a Basler $504 \mathrm{k}$ camera working at $200 \mathrm{~Hz}$, mounted with a Nikkor $105 \mathrm{~mm}$ macro lens with an aperture of f/4. The images were then processed using classic PIV techniques. ${ }^{25}$ Velocity fields were computed using the open source software called MatPIV. ${ }^{26}$ See Ref. 22 for further information on the PIV techniques for trimix-based suspensions.

Figure 4 shows how the flow depth profiles and front position were measured. Four small cameras (Basler A311f) mounted with Fujinon 12.5 mm lenses (Fujinon 12.5 HA-1B), all with an aperture of $\mathrm{f} / 1.4$ measured the flow depth profiles in the $x$ - or $z$-direction. A laser pulse synchronizer (model 610034 manufactured by Berkeley Nucleonics Corporation for TSI) synchronized the laser and the cameras. Images for flow depth profiles were taken at the rate of $28.8 \mathrm{~Hz}$. 


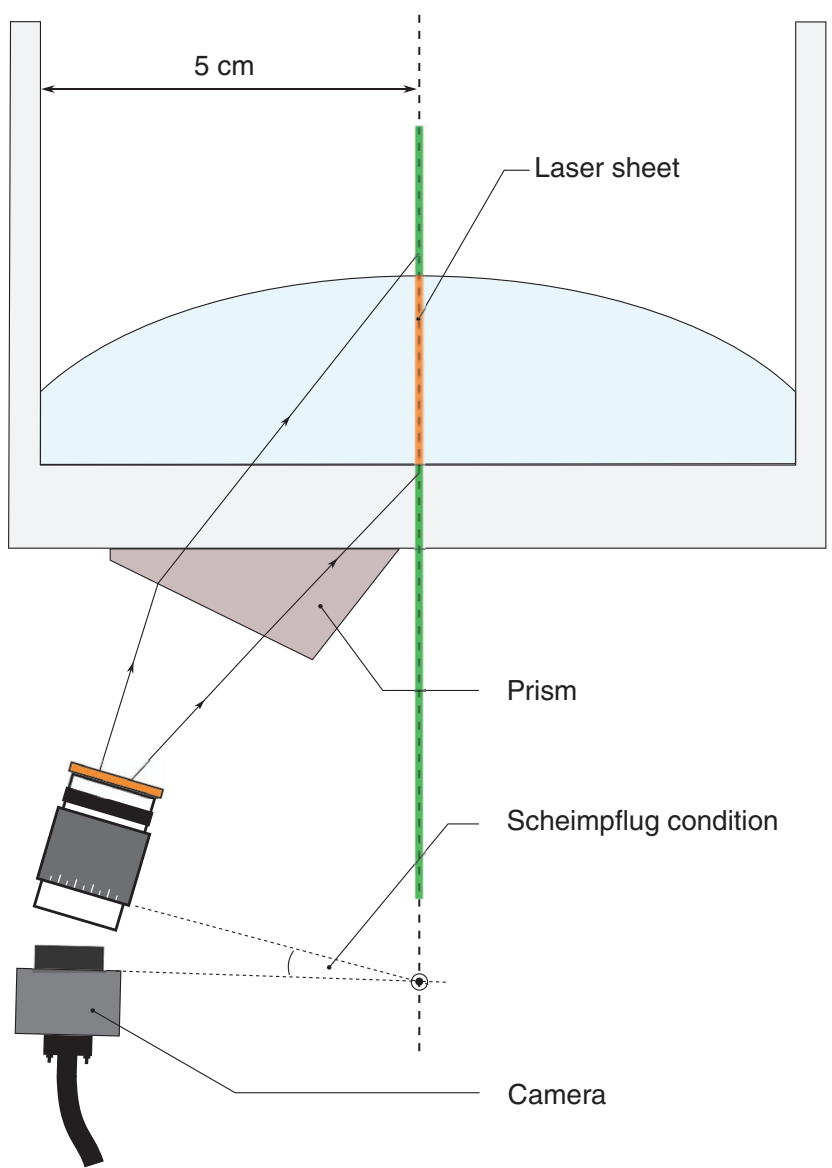

FIG. 3. Sketch of the measurement system for the velocity profiles within the moving suspension. Because of the suspension/air interface and the three-dimensional nature of the flows, we were forced to film the flow from below. The Scheimpflug principle was used to correct perspective distortion caused by the inclination of the lens with respect to the plane viewed by the camera.

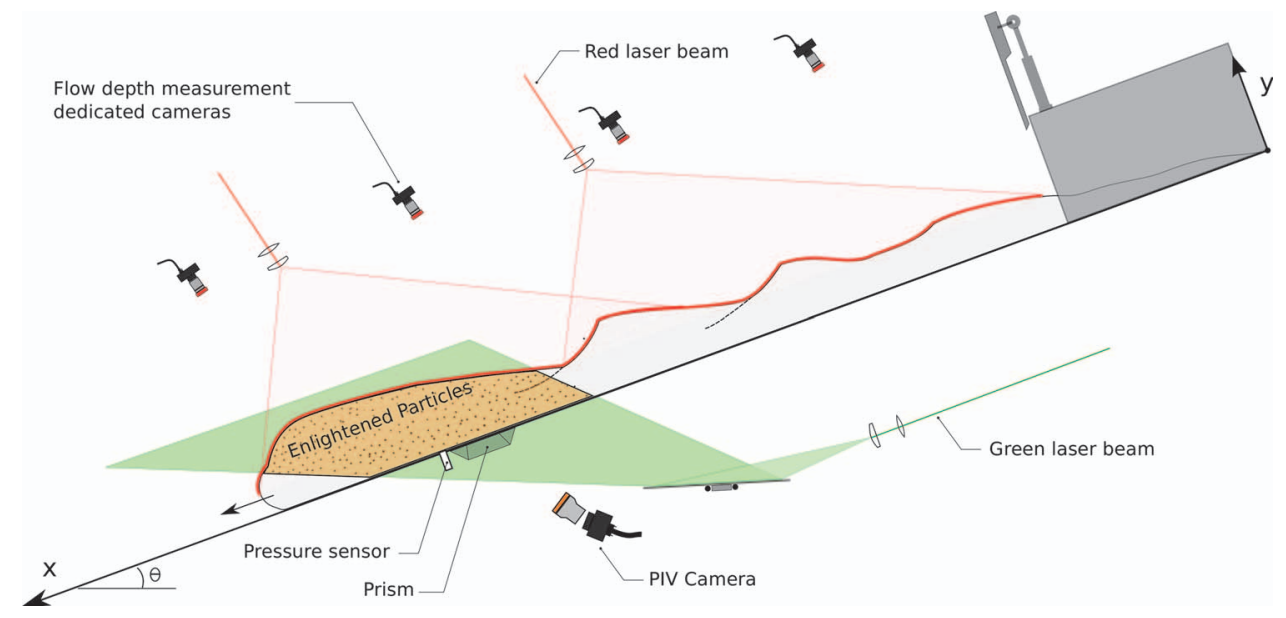

FIG. 4. Schematics of camera and laser positions. The axes are also shown. 


\section{Notation and scaling}

For this flume, we define a three-dimensional Cartesian coordinate system in which the $x$-axis points down the flume, the $y$-axis is in the direction of the upward pointing normal, and the $z$-axis is in the cross-stream direction (see Fig. 2). The upper end of the flume is at $x=0$, while the lower end is at $x=350 \mathrm{~cm}$. The bulk velocity $\mathbf{u}$ has components $u, v$, and $w$ in each of these directions, respectively. $z=0$ refers to the right sidewall (when looking at the flume from the inlet), while $z=W=10 \mathrm{~cm}$ refers to the left sidewall. The front position is denoted by $x_{f}$ and the flow depth by $h(x, z, t)$. The flume inclination is denoted by $\theta\left(\theta=25^{\circ}\right.$ for all runs presented here). The initial volume per unit width is denoted by $A$.

In what follows, we work mostly with non-dimensional flow variables, using hats to distinguish dimensional variables. It is convenient to introduce such non-dimensional variables using scalings that reflect the shallowness of the flow:

$$
\hat{x}=L_{*} x, \quad \hat{y}=H_{*} y, \quad(\hat{u}, \hat{v})=U_{*}(u, \epsilon v), \quad \hat{t}=T_{*} t,
$$

where $H_{*}$ denotes the flow-depth scale, $T_{*}=L_{*} / U_{*}$ is the characteristic time, with $U_{*}$ $=\rho g H_{*}^{2} \sin \theta / \mu$ and $L_{*}$ as the velocity and length scales, respectively, $g$ the gravitational acceleration and $\mu$ the bulk viscosity, and finally $\epsilon=H_{*} / L_{*}$ as the aspect ratio. Due to the possibility that the viscosity may be a flow variable, we keep in mind that in this scaling, $\mu$ refers to the bulk viscosity of a homogeneous suspension. If we use a relation such as the Krieger-Dougherty equation (A3), we should define $\mu$ as $\mu=\mu(\bar{\phi})$, where $\bar{\phi}$ denotes the mean solids fraction. To be consistent with volume conservation, we select the length and depth scales $L_{*}$ and $H_{*}$ such that $L_{*} H_{*}=A, L_{*}=255 \mathrm{~cm}$ being the distance from the flume entrance (or upper end of the reservoir) to the observation window. As emphasis is given to shallow gravity-driven flows of highly viscous fluids on slopes, flows are assumed to be in the limit of high capillary number and low Reynolds and aspect-ratio numbers: $C a=\mu U_{*} / \gamma \gg 1, R e=\rho U_{*} H_{*} / \mu \ll 1$, and $\epsilon \ll 1$.

\section{EXPERIMENTAL RESULTS}

We conducted several experimental campaigns to investigate how the flow dynamics of a fixed volume of suspension was influenced by the flume inclination, initial volume, particle concentration, and density mismatch between the interstitial fluid and particles. ${ }^{23}$ The observed behavior was quite complex and is probably best explained by first describing the typical flow pattern, then by examining each regime of this overall pattern and indicating their distinctive features. This wealth of behaviors, which was observed for minute changes in the flow conditions or material properties, explains why the earlier investigations came to contradictory conclusions regarding the behavior of avalanches of granular suspensions (see Sec. I). For the sake of simplicity, we report the experimental results obtained for a single value of the flume inclination: $\theta=25^{\circ}$. Table I summarizes the different runs presented in this paper. All runs were repeated several times and for some of them, we also slightly changed the boundary conditions (e.g., by wetting the flume bottom with Dibromohexane (DBH) to see if the contact line influenced the front dynamics). A striking point was that all features were reproducible, some of them (such as front position) to within 1\%, others (e.g., the time to observe the stick-slip regime) to within $10 \%-20 \%$.

\section{A. Regime partitioning}

The key parameter that controlled flow behavior was the solids fraction $\bar{\phi}$. For $\bar{\phi} \leq 0.57$, the suspension flowed like a viscous fluid. ${ }^{27}$ In particular, the front position varied with time as $t^{1 / 3}$, consistent with the theory of thin elongating Newtonian flows down a sloping bed ${ }^{28}$ (see also Subsection 2 of the Appendix). For $\bar{\phi} \geq 0.61$, the flow rapidly came to rest. We tried to determine whether a critical value of the solid concentration $\phi_{c}$ (e.g., corresponding to a percolation threshold) controlled the transition from Newtonian to non-Newtonian behavior, but we failed to find a narrow range for $\phi_{c}$. There were several explanations for this. First, the solid concentration was not the sole parameter that controlled the flow dynamics. Second, we used slightly polydisperse particles for 
TABLE I. Features of the different runs: mean solids fraction $\bar{\phi}$, initial mass $m$, particle size distribution, the estimate of the bulk viscosity using the Krieger-Dougherty relation (with $\beta=2$ and $\phi_{m}=0.625$ ), the characteristic flow depth, the velocity and time scales, and the flow Reynolds number. For all runs, the particle density, fluid density, fluid viscosity, and flume inclination were kept constant: $\rho_{f}=\rho_{p}=1.184 \mathrm{~g} / \mathrm{cm}^{3}, \mu_{f}=0.124 \mathrm{~Pa} \mathrm{~s}$, and $\theta=25^{\circ}$. The length scale is $L_{*}=2.55 \mathrm{~m}$.

\begin{tabular}{lcccccccc}
\hline \hline Run & $\bar{\phi}(\%)$ & $m(\mathrm{~g})$ & Distribution & $\mu(\mathrm{Pas})$ & $H_{*}(\mathrm{~m})$ & $U_{*}(\mathrm{~m})$ & $T_{*}(\mathrm{~s})$ & $R e$ \\
\hline A & 57.5 & 6000 & Sieved & 19.4 & 0.020 & 0.100 & 25 & 0.122 \\
B & 58.0 & 6000 & Sieved & 23.9 & 0.020 & 0.081 & 31 & 0.080 \\
C & 58.5 & 6000 & Sieved & 30.3 & 0.020 & 0.064 & 40 & 0.050 \\
D & 59.5 & 6000 & Sieved & 54 & 0.020 & 0.036 & 71 & 0.016 \\
E & 59.5 & 2000 & Raw & 54 & 0.007 & 0.004 & 637 & 0.001 \\
F & 59.5 & 3000 & Raw & 54 & 0.010 & 0.009 & 283 & 0.002 \\
G & 59.5 & 4000 & Raw & 54 & 0.013 & 0.016 & 159 & 0.005 \\
H & 59.5 & 6000 & Raw & 54 & 0.020 & 0.036 & 71 & 0.016 \\
I & 59.5 & 7940 & Raw & 54 & 0.026 & 0.063 & 40 & 0.036 \\
\hline \hline
\end{tabular}

which the definition of critical concentrations (such as the maximum random packing concentration) was more difficult. ${ }^{29-31}$

When the solids fraction was in the $0.575-0.605$ range, we observed three distinct regimes:

- Macro-viscous regime: At early times, the suspension moved downstream like a viscous avalanche in agreement with the observations made, for instance, by Bonnoit et al. ${ }^{16}$ Typically, the front position varies with time as $x_{f} \propto t^{1 / 3}$ and the flow depth profile is closely approximated by the Newtonian profile $h(x, t)=\sqrt{x / t}$. The velocity profiles along the $y$-axis the depth take a parabolic shape, but deviations from this form are possible as a result of particle migration (blunting of the velocity profile) or the free-surface gradient. See Subsection 2 of the Appendix.

- Fracture regime: Before the front reached the end of the flume, the free surface developed undulations as fractures developed along the free surface. These fractures marked the transition to a plastic regime in which the suspension flowed downstream slowly. The flow depth was fairly uniform along the flume. The velocity profiles across the stream showed that all of the shearing was concentrated within a thin layer along the flume bottom.

- Plastic regime: At long times, this regime became unstable and degenerated into a stick-slip regime in which the suspension moved intermittently during episodes of slipping and remained in an arrested state for short periods of time.

In the following, we describe the macro-viscous regime at length. The fracture and plastic regimes are described in Paper II. ${ }^{46}$

\section{B. Macro-viscous regime: Outline}

Among the macroscopic flow features, the front position is of paramount importance not only because it is easy to track, but also because the dependence of $x_{f}$ on $t$ yields insight into the rheological behavior. Figure 5 shows the front position as a function of time in a dimensionless log-log form for all runs. In addition to the experimental data, we have also plotted the similarity solution $x_{f}=(9 t / 4)^{1 / 3}$ (see Subsection 2 of the Appendix for further information). On the whole, the $x_{f}$ $\propto t^{1 / 3}$ scaling offers a proper description of the front evolution, which justifies our reference to these flows as macro-viscous flows. Some departures from the power-law scaling are, however, conspicuous. We observed that for the sieved suspensions at the highest concentrations (Runs B, $\mathrm{C}$, and $\mathrm{D}$ ), the experimental curves diverged from the similarity solution at long times and this departure was even more marked when increasing the particle concentration to $58 \%$ or $59 \%$. For Run D, the flow seemed to come to a halt, but in fact it entered a stick-slip regime, in which the front moved forward intermittently (see Paper II $^{46}$ about the plastic regime). For the unsieved suspensions, the experimental curves lie slightly above the similarity solution, which may be an indication of 


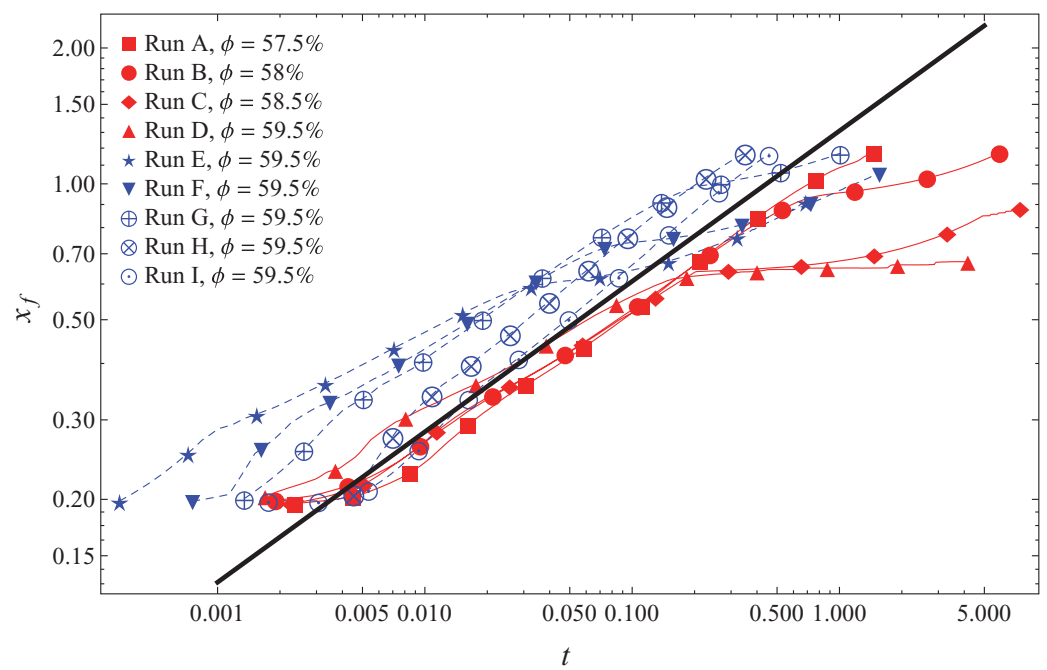

FIG. 5. Position of the front as a function of time for all runs. We also report the similarity solution $x_{f}=(9 t / 4)^{1 / 3}$ (see the Appendix).

particle migration. Indeed, as particles migrate from high to low shear regions, the basal particle concentration decreases and the local viscosity decreases, leading to higher velocities.

As shown by Fig. 5, all $x_{f}(t)$ curves were first characterized by a power-law behavior $x_{f}(t)=\lambda t^{p}$ (showed as a "linear" behavior in the log-log representation) at short and intermediate times, then for $t>t_{c}$ (with $t_{c}$ a critical time) they flattened out or became convex. Note that this transition was abrupt and was reflected by a kink in the $x_{f}(t)$ curves at $t \approx t_{c}$. Table II reports the values of the parameters $\lambda$ and $p$ adjusted on the data together with an estimate of the critical time $t_{c}$. Deviations from the Newtonian law $x_{f}=(9 t / 4)^{1 / 3}$ may result from particle migration. A crude way of accounting for particle migration in the constitutive equation is to assume that the bulk rheological behavior can be captured by a power-law model (even though the behavior is locally linear):

$$
\Sigma_{x y}=\mu_{f} n_{s}(\phi) \dot{\gamma}=\kappa \dot{\gamma}^{n},
$$

where $\Sigma_{x y}$ denotes the shear stress, $n$ an index ( $n \leq 1$ in most cases), and $\kappa$ the dimensionless consistency (see Subsection 3 of the Appendix). Adopting this rheological equation in place of the Newtonian law and assuming that $n$ is constant, we can derive a new expression for the front position with time, which is given by Eq. (A13). Making use of this relation and adjusting it on the $x_{f}$ data, we can deduce the value of $n$. As shown by Table II, most runs were characterized by $n<$ 1 , signifying a shear-thinning behavior, but for Runs $\mathrm{H}$ and I, we found $n>1$, which would mean

TABLE II. Values of the parameters $\lambda$ and $p$. Using Eq. (A13), we inferred the value of $n$. $t_{c}$ was estimated as the time until which the front position varied with time like $x_{f}(t)=\lambda t^{p}$.

\begin{tabular}{lcccc}
\hline \hline Run & $\lambda$ & $p$ & $n$ & $t_{c}$ \\
\hline A & 1.12 & 0.33 & 0.98 & 1.05 \\
B & 1.05 & 0.29 & 0.71 & 0.94 \\
C & 1.00 & 0.28 & 0.65 & 0.64 \\
D & 1.05 & 0.27 & 0.58 & 0.15 \\
E & 1.35 & 0.23 & 0.42 & 0.03 \\
F & 1.54 & 0.28 & 0.62 & 0.06 \\
G & 1.76 & 0.32 & 0.86 & 0.20 \\
H & 1.82 & 0.37 & 1.47 & $\cdots$ \\
I & 1.59 & 0.38 & 1.59 & $\cdots$ \\
\hline \hline
\end{tabular}


that the suspension behaved like a shear-thickening fluid. Flow visualization showed, nevertheless, that this was not the case (see below, e.g., Fig. 7). This implies that (2) is just an empirical way of describing the departure from the Newtonian law (due to the blunting of the velocity profile), but cannot be used as a constitutive equation for the suspension (as $n$ varies within the flow).

The initial mass of the suspension had a strong influence on the subsequent motion of the granular suspension. For the avalanche releasing the largest mass of suspension $(m=7.94 \mathrm{~kg}$, Run I), the front position quickly tended to the asymptotic curve $x_{f} \propto t^{1 / 3}$, but at long times, the front accelerated slightly relative to the Newtonian case. For both sieved and unsieved suspensions with mass $m=6 \mathrm{~kg}$ (Runs A-D, H), the front behavior was very similar at early times $(t<0.01)$, but at later times $(0.01<t<0.02)$, the data pertaining to the sieved suspensions collapsed on a single curve, while the unsieved suspension (Run H) moved faster than the Newtonian case. For the smallest avalanche ( $m=3 \mathrm{~kg}$, Run F), the time variations in the front position showed that at the shortest times $\left(t \leq 2 \times 10^{-3}\right)$, the front position scaled as $x_{f} \propto t^{0.4}$ (slumping of the mass after the lockgate removal). At intermediate times $(0.002<t<0.06)$, the front position varied like $x_{f} \propto t^{0.28}$ rather than $x_{f} \propto t^{1 / 3}$, but the front velocity was $50 \%$ higher than that of a homogeneous Newtonian fluid. At longer times, the curve for the front position flattened out, implying that the mass suddenly slowed down. To summarize, we observed that the larger the initial mass, the closer the front position to the Newtonian law. This behavior was qualitatively consistent with the theoretical analysis of the characteristic times of particle migration ${ }^{32}$ (e.g., the typical time to reach steady state for suspensions experiencing particle migration). As summarized in the Appendix, the characteristic time of particle migration is proportional to the typical flow depth $H_{*}^{3}$, hence to the volume $A^{3}$ (since the characteristic length $L_{*}$ was kept constant). If we interpret the kink observed in the $x_{f}(t)$ curve as the hallmark of the steady regime (see Subsection 3 of the Appendix), we then find that multiplying the mass by a factor of 2 (Runs E-G) should lead to an eight-fold increase in the steady-state time. Yet, experimentally, the change in behavior occurred at $t_{c} \sim 0.03$ for Run E compared with $t_{c} \sim 0.2$ for Run G, that is, a factor of 7 faster, which is close to the theoretical ratio. Between Runs E and H, the mass was tripled, leading to a time ratio of 27 , which may explain why we did not observe any change in the $x_{f}(t)$ curves for the largest masses (Runs H and I).

From the observing window located at $x=1$, we measured the evolution of the depth profile and by taking images from below, we recorded the velocity profiles at different times. To illustrate the influence of the particle size distribution on the velocity profiles, we examined the experimental data corresponding to Runs A and $\mathrm{H}$ simultaneously, as they had the same flume inclination $\left(\theta=25^{\circ}\right)$ and the same initial mass $(6 \mathrm{~kg}$ ). We did not compare Run $\mathrm{H}$ with Run $\mathrm{D}$ (which would have seemed more natural as they had the same solids fraction) since for the latter, the suspension moved downwards as a rigid block, with all the shear localized within a very thin layer (3 particle diameters) along the flume bottom (all velocity profiles were then uniform).

The respective upper panels of Figs. 6 and 7 show the experimental flow-depth evolution at $x=1$ and the theoretical $h$ profile (zeroth-order solution provided by lubrication theory, see (A9) in the Appendix). The experimental and theoretical flow-depth curves $h(1, t)$ did not start at the same time due to the differences in the front position evolution between theory and observation. Therefore, we shifted all of the $h$ curves slightly so that they had the same time origin. In all of these plots, $\Delta t$ $=t-t_{0}$ is the time elapsed since the front passed $x=1$, where $t_{0}$ refers to the arrival of the front at $x=1\left(t_{0}=4 / 9\right.$ for lubrication theory, see Subsection 2 of the Appendix). In the lower panels, we plotted the experimental velocity profiles (dots) and the theoretical Newtonian velocity profile (A5) in dimensionless form. To plot these profiles, we used the bulk viscosity value noted in Table I. We neglected the influence of the free-surface gradient, which may alter the amplitude of the velocity in high-curvature regions (the leading edge). One reason for this was that it was not always easy to estimate the free-surface gradient owing to the numerous bumps that distorted the free surface. Note also that for all velocity profiles, we used a scaled coordinate $y / h$ to facilitate the comparison of results. We noticed that a power-law velocity profile captured the data well, so we also obtained the velocity profiles using a power-law rheological model, (2), of the form

$$
u(x, y, t)=\frac{n \kappa^{1 / n}}{n+1}\left(h^{1+1 / n}-(h-y)^{1+1 / n}\right) .
$$



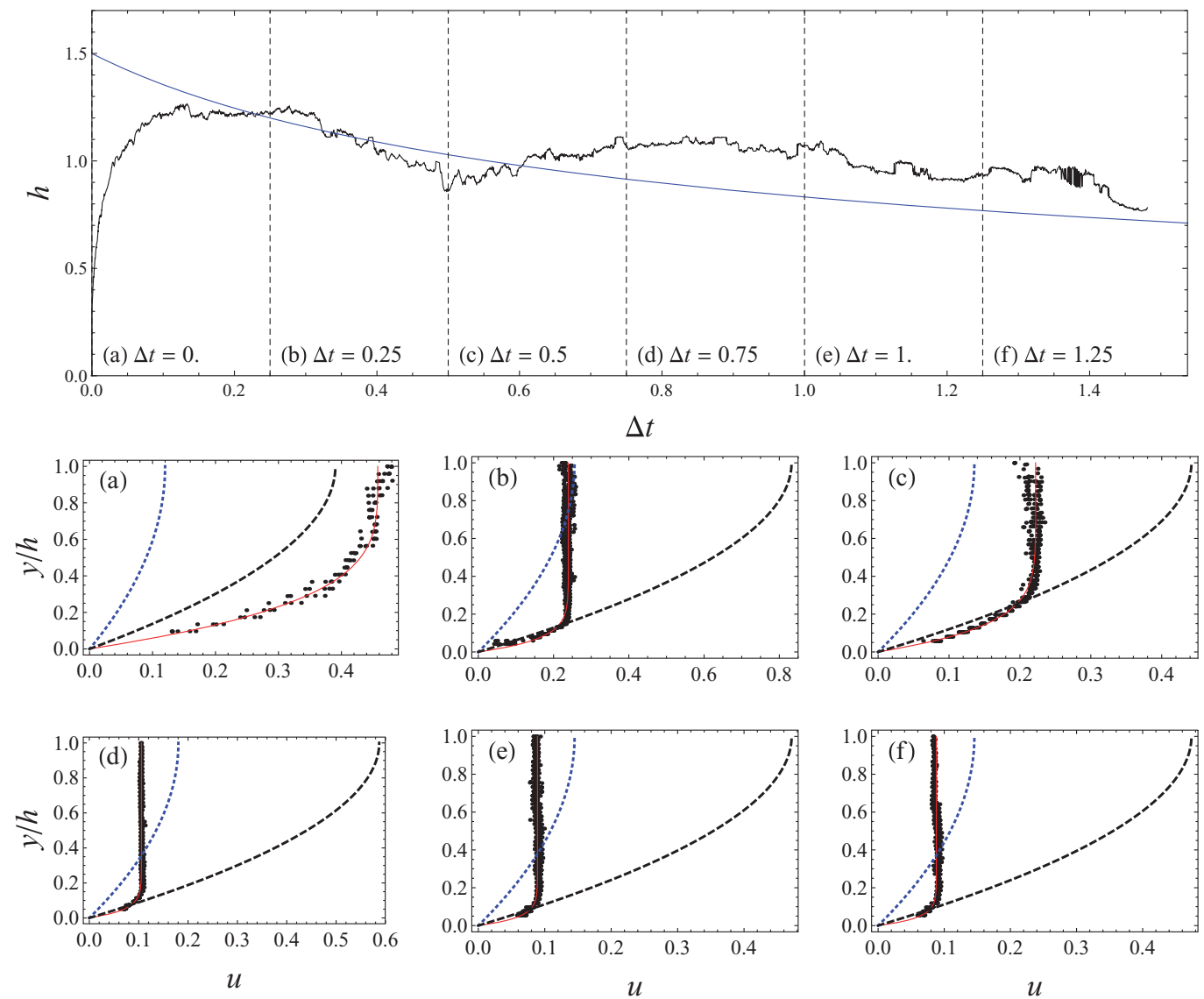

FIG. 6. Flow depth evolution $h(x, t)$ at $x=1$ and velocity profiles taken at different times $\Delta t$ after the front passed, for Run A $(\bar{\phi}=0.575$, sieved). In the upper panel, we plotted the experimental data (black line) and the theoretical solution $h(x, t)$ given by lubrication theory (A9) (blue line). In the lower panels, we have plotted the velocity profiles: the dots represent the experimental data, the solid (red) line is the power-law function (3), the dashed line shows the Newtonian profile (A5) when the Krieger-Dougherty equation (A3) is used for the bulk viscosity, and the dotted line shows the Newtonian profile (A5) when Eq. (A4) proposed by Zarraga, Hill, and Leighton ${ }^{34}$ is used for the bulk viscosity. The fitted values of $n$ and $\kappa$ are the following ones: (a) $n=0.327$ and $\kappa=53.4$, (b) $n=0.073$ and $\kappa=104.4$, (c) $n=0.160$ and $\kappa=64.7$, (d) $n=0.049$ and $\kappa$ $=93.8$, (e) $n=0.044$ and $\kappa=82.9$, and (f) $n=0.042$ and $\kappa=83.6$.

No slipping at the bottom was detected, and therefore, the streamwise velocity satisfied the no-slip condition, usual for Newtonian fluids, but not generally exhibited by concentrated suspensions. ${ }^{33}$ Essentially, Eq. (3) was used to obtain a measure of the deviation from the Newtonian case, which gave information on the degree of blunting of the velocity field due to migration (see Subsection 3 of the Appendix, where the effects of particle migration are quantified using $n$ ).

For the sieved suspension at the lowest concentration (Run A, $\phi=57.5 \%$ ), the flow depth profile was crudely captured by the theoretical profile (A9), as shown by the upper panel of Figure 6, but the free surface exhibited bumps and irregularities, which were not seen at lower solids fractions. Except for subplot (a), showing data taken from just behind the front, the velocity profiles in Figure 6 were not parabolic and did not match the theoretical profiles (A5) corresponding to the flow of a homogeneous Newtonian fluid. Interestingly, the theoretical profile matched the experimental velocity profile in the basal layer $(y / h<0.1)$ when the Krieger-Dougherty equation (A3) was used. Other equations such as (A4) provided poor agreement. For all times, we successfully fitted the parameters $n$ and $\kappa$ to the velocity data using the method of least squares. Their respective values are reported in the caption. There was a sudden drop from an intermediate value, $n \sim 0.5$, at the leading edge to a low value, $n \sim 0.04$, in the body. Note that $n$ seemed to depend on the free-surface gradient instead of time (as expected from particle migration theory): for subplots (a) and (c), this gradient 

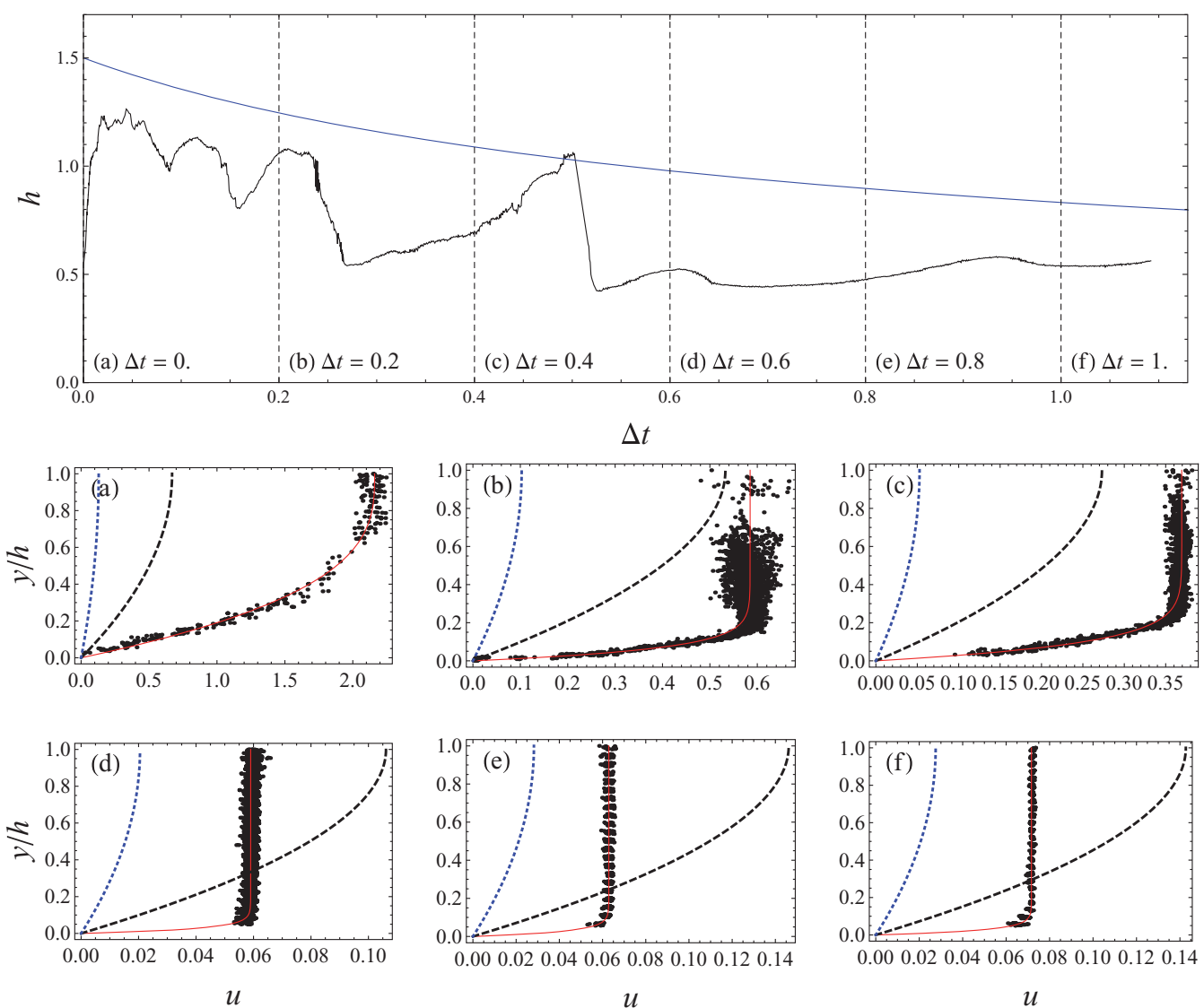

FIG. 7. Flow depth evolution $h(x, t)$ at $x=1$ and velocity profiles taken at different times $\Delta t$ after the front passed, for Run $\mathrm{H}(\bar{\phi}=0.595$, unsieved). In the upper panel, we plotted the experimental data (black line) and the theoretical solution $h(x$, t) given by lubrication theory (A9) (blue line). In the lower panels, we have plotted the velocity profiles: the dots represent the experimental data, the solid (red) line is the power-law function (3), the dashed line shows the Newtonian profile (A5), and the dotted (blue) line shows the Newtonian profile (A5) when Eq. (A4) proposed by Zarraga, Hill, and Leighton ${ }^{34}$ is used for the bulk viscosity. The fitted values of $n$ and $\kappa$ are the following ones: (a) $n=0.515$ and $\kappa=41.9$, (b) $n=0.069$ and $\kappa=82.2$, (c) $n=0.102$ and $\kappa=58.3$, (d) $n=0.021$ and $\kappa=43.9$, (e) $n=0.026$ and $\kappa=53.7$, and (f) $n=0.025$ and $\kappa=46.5$.

dependence was much more pronounced than for other subplots and the $n$ value (respectively, 0.32 and 0.16) differed from the value found for the body $(n \sim 0.04)$.

Figure 7 shows the same information for Run $\mathrm{H}$ (unsieved suspension). On the whole, the flow pattern looked very similar, but there were three striking points that deserve further attention. First, the measured flow depth was significantly smaller than the theoretical profile and exhibited bumps in the region between the front and the body, whose amplitude was in some cases as high as the body flow depth(the body free-surface was much smoother). Second, even with solids fractions as large as 0.595 , part of the flow was sheared, whereas for the sieved suspensions at the same concentration, the flows did not exhibit any basal shear and flowed like sliding blocks. Third, the Newtonian approximation underestimated the velocities up to a factor of 4 close to the leading edge. Away from the front (typically for $t>0.5$ ), the order of magnitude predicted by Newtonian theory was correct, but the shape of the velocity profile was not properly captured.

\section{Further analysis: Run I}

Here we present additional results for Run I, which corresponds to the largest mass tested (nearly $8 \mathrm{~kg}$ ). As the flow depth was larger than for other runs and the flow duration longer, it was 


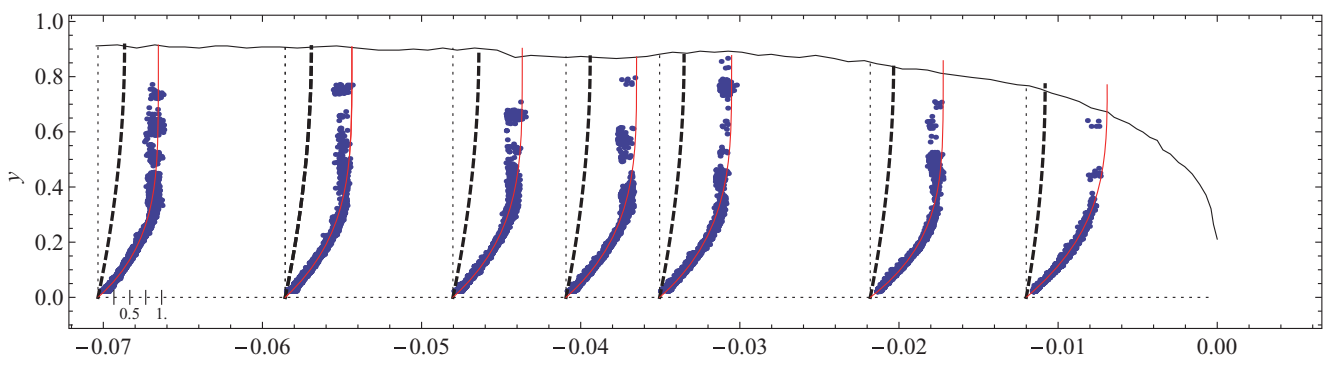

(a)

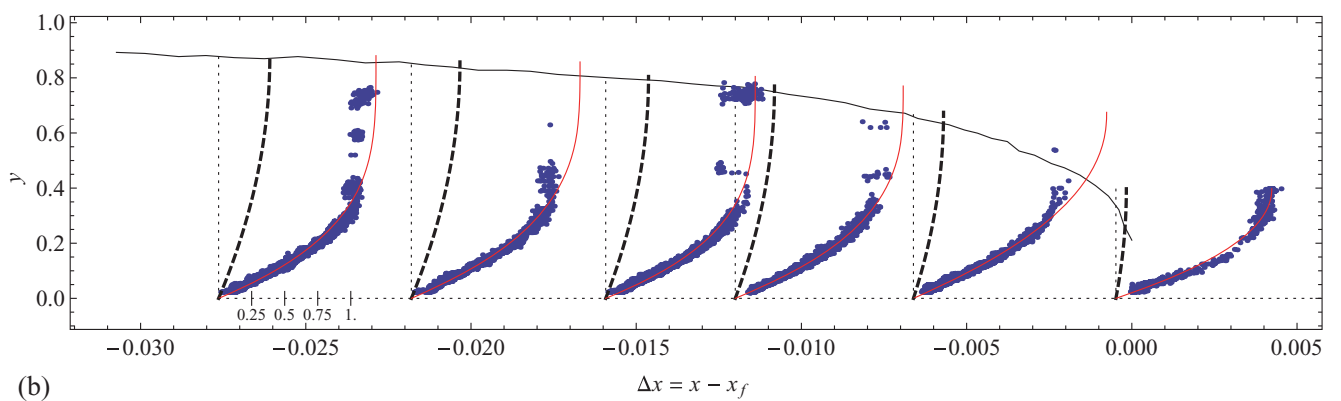

FIG. 8. Velocity field at $t=0.26$ within the body (a) and close-up view of the velocity profiles within the leading edge (b) for Run I ( $\bar{\phi}=0.595$, unsieved). The dots represent the velocities measured within the flowing suspension at the middle of the flume ( $5 \mathrm{~cm}$ from the sidewall), while the dashed (black) line represents the Newtonian model with $\mu=54 \mathrm{~Pa} \mathrm{~s}$ (value given by the Krieger-Dougherty relation (A3) with $\beta=2$ and $\phi_{m}=0.625$ ). The solid (red) line is the power-law function (3) adjusted on the data.

easier to make measurements for this mass. Figures $8(a)$ and $8($ b) show the velocity field (streamwise component) within the body and the leading edge, respectively, taken at $t=0.26$. In addition to the experimental velocities, we plotted the theoretical profiles corresponding to steady-state Newtonian flows, given by Eq. (3) with $\mu=54 \mathrm{~Pa} \mathrm{~s}$, the value given by the Krieger-Dougherty relation (A3) (with $\beta=2$ and $\phi_{m}=0.625$ ). We also compared the empirical velocity profiles obtained by adjusting the power-law function (3) to the data. We retrieved similar observations as beforehand (for Run $\mathrm{H}$ ): the experimental velocity profiles departed significantly from the parabolic Newtonian profile, but were well captured by the power-law model (3). Note that the velocity profiles were more blunt in the body than in the tip region. The general impression was that the blunting decreased with increasing free-surface gradient. This contrasted with the theoretical description of particle migration as a time-dependent diffusive process, in which the particles migrate from high- to low-shear regions over time. One possible explanation for this discrepancy between theory and observation is, as in segregating granular flows, ${ }^{35}$ that there was a recirculation cell located within the head, which caused the particles to mix, making the suspension more locally homogeneous.

Figure 9 shows the flow-depth profiles at different times together with the theoretical profiles. At least qualitatively, the theoretical profiles looked like the measured profiles, which confirms once again that the Newtonian model roughly captured the bulk behavior. Taking a closer look at the differences between computed and observed profiles reveals two interesting features. First, at long times (here for $t \geq 0.2$ ), the profiles were increasingly bumpy, the free surface exhibiting a series of "waves." Second, while the flow depth profile within the body varied as the square root of the distance, as expected from Eq. (A9), the front was blunter than the theory predicted. As explained in Paper II, ${ }^{46}$ the development of waves on a free surface was the consequence of fractures that grew in size in the course of motion, but the exact mechanism remains unknown.

An interesting feature of free-surface flows down chutes is that the cross-stream flow depth profile provides information on the existence of non-zero normal stress differences. ${ }^{36,37}$ For instance, 

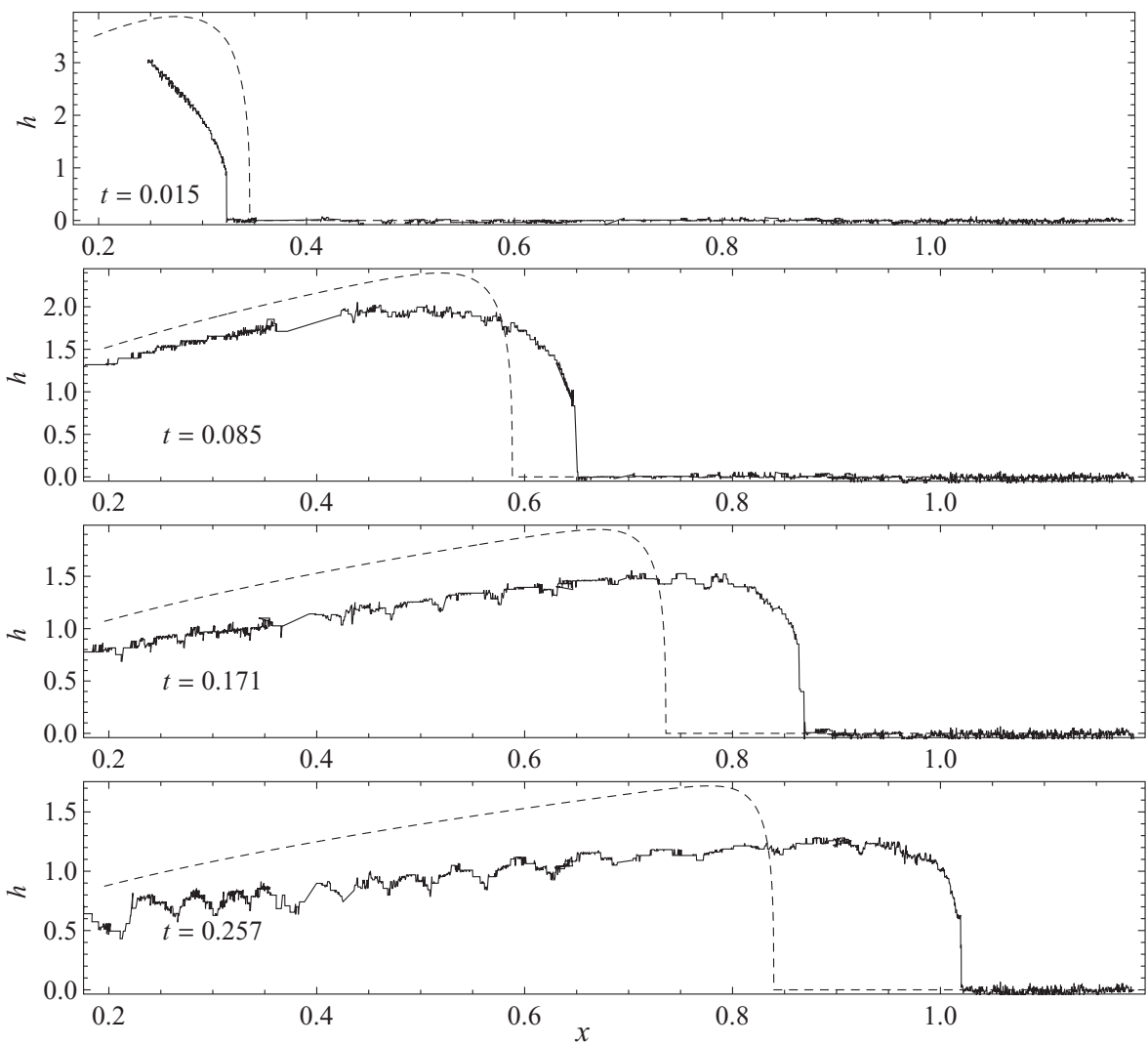

FIG. 9. Longitudinal flow depth profiles at times $t=0.015,0.085,0.171$, and 0.257 for Run $\mathrm{I}(\bar{\phi}=0.595$, unsieved). The solid lines represent the raw data, while the dashed lines show the analytical solution for a homogeneous Newtonian fluid (with an estimated bulk viscosity of $\mu=54 \mathrm{~Pa} \mathrm{~s}$ ). The experimental flow depth profiles were not filtered. Note that these profiles were obtained by stitching images from different cameras, which explains why the point density was not uniform along the flume; stitching gave rise to profiles that were locally smoother than in reality whenever the overlap region was not sufficiently long. For the theoretical flow depth, we used the composite solution given by Ancey, Cochard, and Andreini ${ }^{28}$ (Eq. (A9) is merely the outer solution).

Tanner $^{36}$ showed that in the absence of a normal stress difference, i.e., $N_{2}=\Sigma_{y y}-\Sigma_{z z}=0$ (which is the case for Newtonian fluids), the free surface is flat. When $N_{2}$ is non-zero, the free surface is curved; to leading order, the cross-stream profile is

$$
\rho g h(x, z) \cos \theta=c+N_{2}\left(\dot{\gamma}_{z}\right),
$$

with $\dot{\gamma}_{z}=d u(z, h) / d z$ the shear rate in the $z$-direction and $c$ a constant of integration. Symmetry implies that this shear rate and the second normal stress difference $N_{2}$ must vanish at the centerline $(z=W / 2)$. When $N_{2}<0$, the free surface should bulge upward and when $N_{2}>0$, the depth profile reaches its minimum at the centerline.

Figure 10 shows the flow-depth profiles in the $z$-direction at different times (nearly the same as for Fig. 9). At early times, the profile was convex, which provided evidence that the material exhibited non-zero normal stress differences or, more specifically, the second normal stress difference $N_{2}$ was negative as observed in other flow configurations. ${ }^{34,37}$ At later times, the free surface flattened out, which may be interpreted as a significant decrease in the second normal stress difference. Even when the free surface became uneven (for $t \geq 0.25$ ) with wave amplitude exceeding $50 \%$ of the mean flow depth, the cross-stream profile remained fairly flat. 

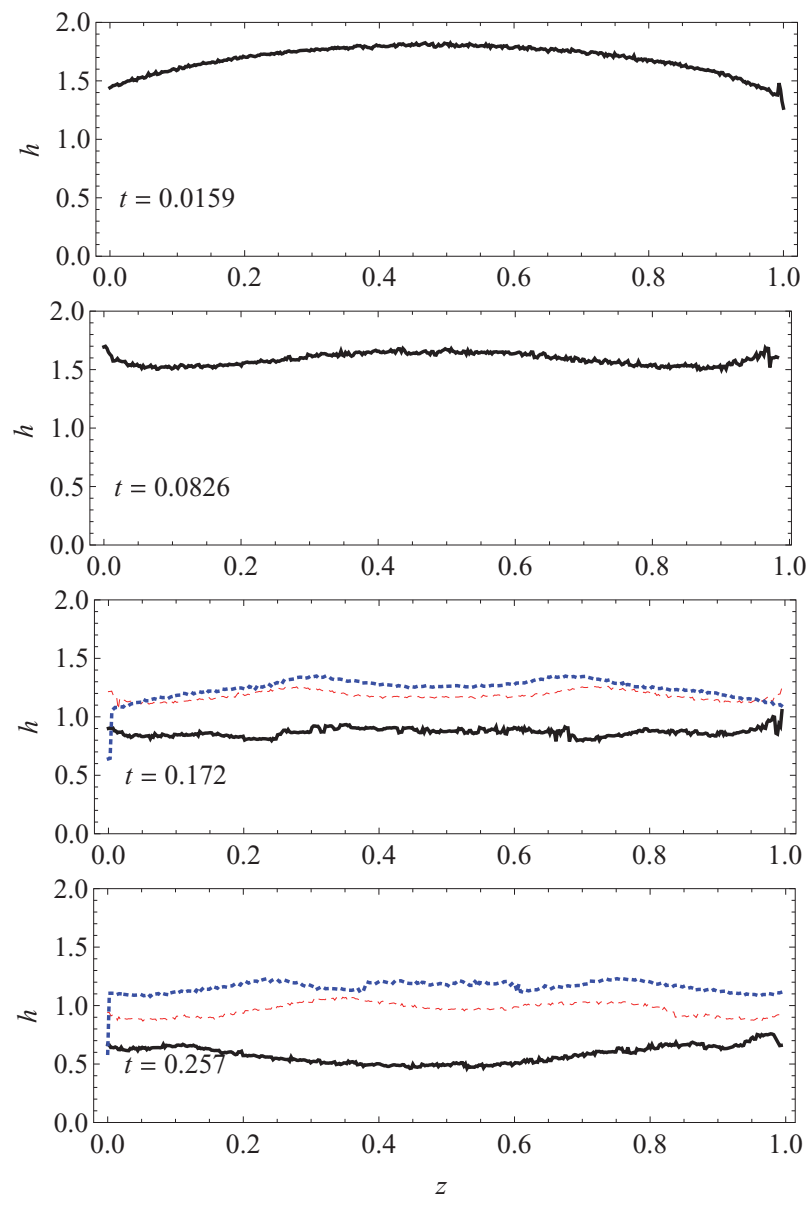

FIG. 10. Cross-stream flow depth profiles at times $t=0.015,0.085,0.171$, and 0.257 for Run $\mathrm{I}(\bar{\phi}=0.595$, unsieved $)$. The profiles were measured across the flume at $x=0.26(\hat{x}=66 \mathrm{~cm}$, solid line), $x=0.5(\hat{x}=127 \mathrm{~cm}$, red dashed line), and $x$ $=0.8(\hat{x}=205 \mathrm{~cm}$, blue dotted line $)$. Same experimental conditions as for Fig. 9 .

\section{CONCLUDING REMARKS}

As far as we are aware, this paper is the first to describe the internal dynamics of highly concentrated particle suspensions under time-dependent flow conditions (the so-called dam-break problem) by investigating both macroscopic properties (front position over time, flow depth profile) and microscopic features (velocity fields within the flowing suspension). We failed to measure local particle concentrations with sufficient accuracy.

The overriding result was that the bulk behavior was much more complicated than previously reported. At short times $(t<0.2-0.5)$, the flow behavior looked like that of a homogeneous Newtonian fluid. More specifically, the front moved as $x_{f} \propto t^{1 / 3}$ and the flow depth profile matched the Newtonian profile fairly well. Close to the front, the velocity profiles were parabolic. There was also clear evidence of non-Newtonian behavior: bulging of the free surface, which was the hallmark of normal stress effects (with $N_{2}<0$ ), and blunting of the velocity profiles near the free surface, which was likely to have arisen from particle migration away from the bottom. We referred to this regime as the macro-viscous regime. At longer times, the free surface became more and more bumpy as the bulk fractured (see Paper $\mathrm{II}^{46}$ ). Eventually, the granular suspension moved intermittently: episodes of slipping were followed by short periods of rest.

The most recent experimental investigations into the rheological behavior of granular suspensions comprising neutrally buoyant particles ${ }^{16,17,21,38,39}$ have led to the hypothesis that the 
continuous fluid phase imparts most of its properties to the bulk, that is, the stresses are linearly dependent on the shear rate and the disperse solid phase does not play a significant role in the rheological response except to generate normal stress differences and enhance energy dissipation. In the limit of high concentrations, the suspension approaches a jammed solid state, which is characterized by the formation of load-bearing force chains throughout large clusters or particle networks. From this perspective, non-Newtonian behavior results from the increase in the correlation length of mutually interacting particles.

In many respects, our measurements confirmed that highly concentrated suspensions exhibited macro-viscous behavior: the front position, depth profile, and velocity profiles within the head were in overall agreement with those observed with Newtonian fluids and the departures from this behavior (bulging of the free surface and blunting of the velocity profile) were reasonably well explained by microstructural theories of particle suspensions. Unsurprisingly, we saw the same behavior as that observed by Ward et al. ${ }^{17}$ and Bonnoit et al. ${ }^{16,39}$ for suspensions with concentrations of $\phi \leq 0.56$. Yet, at higher concentrations, typically here for $\phi>0.57$, the flow pattern observed in our flume was markedly different from the observations reported by these authors. More specifically, Bonnoit et al. ${ }^{16}$ stated that the suspension behaved like a homogeneous Newtonian fluid, with little evidence of particle migration. Bonnoit et al..$^{39}$ demonstrated that there was a critical depth, $\xi \approx 0.2\left(\mu^{2}(\phi) /\left(\rho^{2} g\right)\right)^{1 / 3}$, below which the bulk viscosity increased and became scale-dependent, and above which it matched the Krieger-Dougherty viscosity. Our results provided evidence that particle migration occurred, but its effects were mitigated within the leading edge, probably because of a recirculation cell in the tip region, which acted to homogenize the suspension. All of the $x_{f}(t)$ curves deviated from the Newtonian curve $x(t)=(9 t / 4)^{1 / 3}$ to a varying degree and at a critical time there was a rapid transition to another regime. The depth at which this transition occurred was systematically larger than the critical depth found by Bonnoit et al. ${ }^{39}$ but their respective values were close to each other: it occurred for $h$ between 2 and $3 \mathrm{~cm}$, with $\xi$ ranging from 0.6 to $1.2 \mathrm{~cm}$ when the solids fraction was increased from 0.575 to 0.595 . Importantly, we observed the transition to more complicated flow regimes (the fracture and plastic regimes), which was not observed by others (see Paper $\left.\mathrm{II}^{46}\right)$.

Our velocity profiles were reminiscent of the plug flows observed with plastic flows. We can therefore ask whether this reflects a Coulomb behavior at the bulk scale, which would result from frictional contacts at the particle scale. Given the very high precision reached in the density control of the fluid and particles (relative error lower than $0.05 \%$ ), we found it questionable that sedimentation occurred in our experiments and resulted in Coulomb behavior (which, admittedly, would offer a short practical explanation for the shape of the velocity profiles and the arrested state seen with some of our runs). Authors such as Fall et al. ${ }^{21}$ and Brown and Jaeger ${ }^{40}$ have provided evidence that solid-like properties (e.g., existence of a yield stress) arise from the finite density difference between the particles and fluid, but they also pinpointed the role of surface tension in the appearance of an artificial yield stress. According to these authors, for suspensions composed of $100 \mu \mathrm{m}$ particles, an apparent yield stress of $\tau_{c} \sim 100 \mathrm{~Pa}$ arises when their solids fraction comes close to the maximum concentration. If we interpret the blunt velocity profiles as typical of a plug flow, we find a yield stress $\tau_{c} \sim 65 \mathrm{~Pa}$ for Run I, a value reasonably close to the previous estimate. Yet, such blunt profiles were also observed for solids fractions as low as $52 \%$ and were thus not a distinctive feature of highly concentrated suspensions. Another possibility lies in the development of weak adhesion forces, as has been reported by Snabre and Pouligny ${ }^{41}$ with PMMA particles. The fracture and plastic regimes would have resulted from the appearance of cohesive forces within the bulk as particles migrated and became more densely packed. We carried out additional tests (Couette cell, slump tests) that did not reveal any plastic behavior induced by cohesive forces. We also changed the fluid composition by using only UCON oils (the suspension was no longer transparent) and repeated the dam-break experiments without observing significant changes in the overall flow pattern (specifically the transition to a plastic regime).

Another explanation for this apparent plastic behavior lies in the existence of a critical shear rate $\dot{\gamma}_{c}(\bar{\phi})$ and the occurrence of a viscosity bifurcation. ${ }^{42}$ Ovarlez, Bertrand, and Rodts ${ }^{20}$ have proposed 

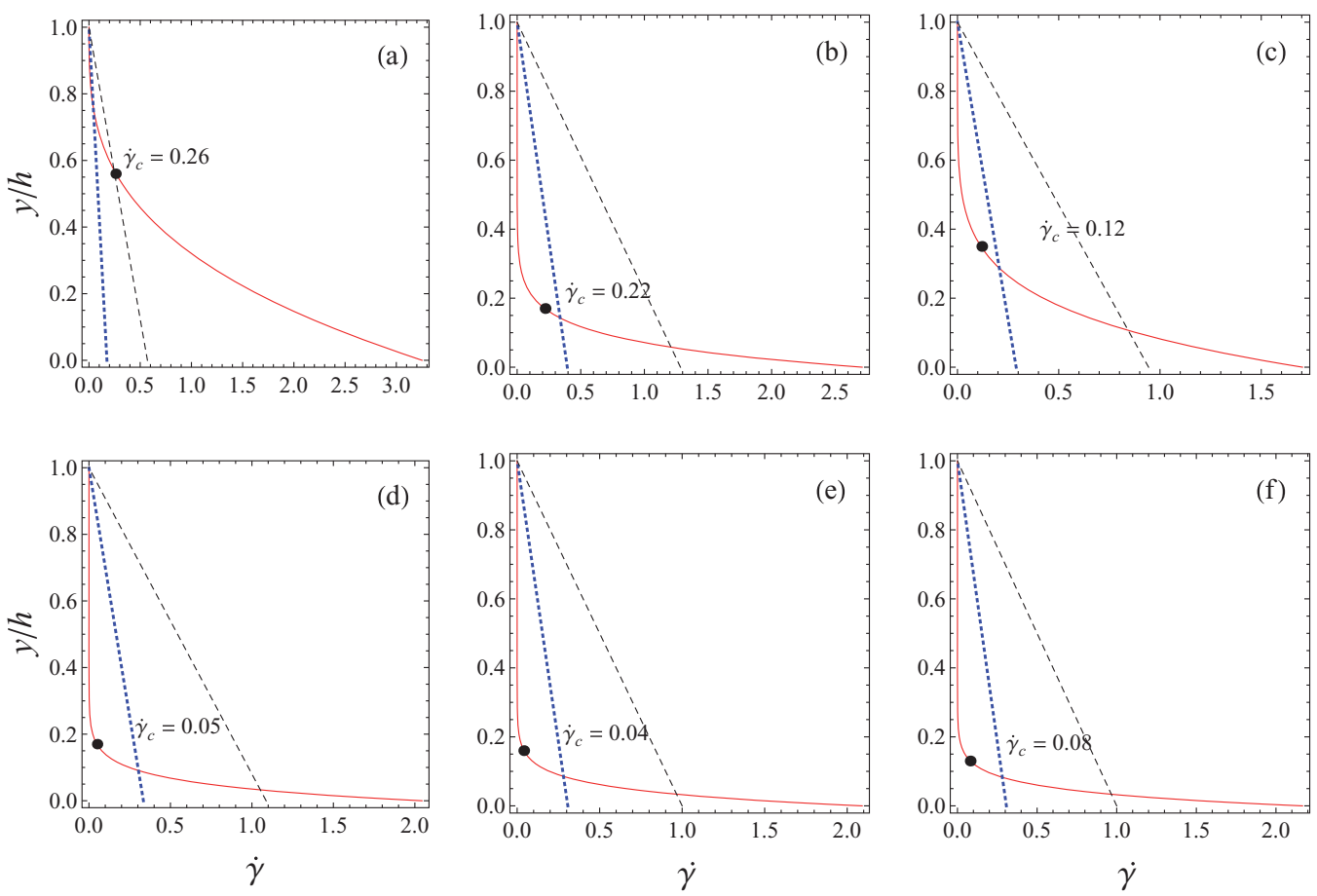

FIG. 11. Shear rate profiles for Run A ( $\bar{\phi}=0.575$, sieved). The solid line shows the shear rate $\dot{\gamma}$ obtained by differentiating the power-law function (3) fitted on the experimental data. The dashed (black) line represents the theoretical shear rate $\dot{\gamma}=\Sigma_{x y} / \mu(\bar{\phi})$ with $\mu(\bar{\phi})$ the Krieger viscosity function (A3). The dotted (blue) line represents the shear rate $\dot{\gamma}=\Sigma_{x y} / \mu(\bar{\phi})$ with $\mu(\bar{\phi})$ the Zarraga viscosity function (A4). The critical shear rate is also reported.

the following very simple model to describe the flow behavior of a granular suspension:

$$
\dot{\gamma}>\dot{\gamma}_{c}(\bar{\phi}) \Rightarrow \Sigma_{x y}=\mu(\phi) \dot{\gamma},
$$

and when $\dot{\gamma} \leq \dot{\gamma}_{c}(\bar{\phi})$, the material stops flowing and the solids fraction is in excess of $\phi_{m}$ (as an effect of particle migration). To test this model, we computed the experimental and theoretical shearrate profiles. The theoretical profile was obtained by assuming that the concentration profile was uniform $(\phi(y)=\bar{\phi})$ and $\dot{\gamma}=\Sigma_{x y} / \mu(\bar{\phi})$, with $\Sigma_{x y}$ given by (A10) and $\mu(\bar{\phi})$ an empirical viscosity function such as (A3) or (A4). Figures 11 and 12 show the corresponding profiles for Runs A and $\mathrm{H}$, respectively. First note that for both runs, the theoretical and experimental profiles were radically different, which means that the assumption of a uniform concentration profile is incorrect (independently of the viscosity function chosen). As the experimental shear rate was computed by differentiating the power-law function (3) that was fitted on the data, it tended continuously to zero away from the bottom and therefore, from this perspective, there was no critical shear rate. However, the velocity data have a fair amount of scatter around an apparently constant value (the free-surface velocity), which prevents a conclusive interpretation. Indeed, even though the upper layers seemed to be slightly sheared (to the naked eye and statistically), the resulting shear rate was often lower than the standard error made in its computation, which means that the assumption of zero shear rate in the upper layers was admissible. Thus, we defined a critical shear rate $\gamma_{c}(\bar{\phi})$ by first determining the depth at which the experimental velocity profile (see Figs. 11 and 12) became uniform, then evaluating the shear rate at this point by differentiating (3). For Run A, we found $\dot{\gamma}_{c}=0.08 \pm 0.03$ $\left(\hat{\gamma}_{c}=0.40 \pm 0.15 \mathrm{~s}^{-1}\right.$ in a dimensional form), while for Run $\mathrm{H}$, the estimate was less accurate $\dot{\gamma}_{c}=0.18 \pm 0.14\left(\hat{\gamma}_{c}=0.36 \pm 0.25 \mathrm{~s}^{-1}\right)$. Interestingly, the former estimate was close to the value found by Huang et al. ${ }^{42}$ with a solids fraction $\bar{\phi}=0.58$. Note also that for Run A, the relative success of the Krieger-Dougherty equation in capturing the velocities in the basal layer (see Fig. 6) is due to its having provided the correct magnitude of the shear rate. 

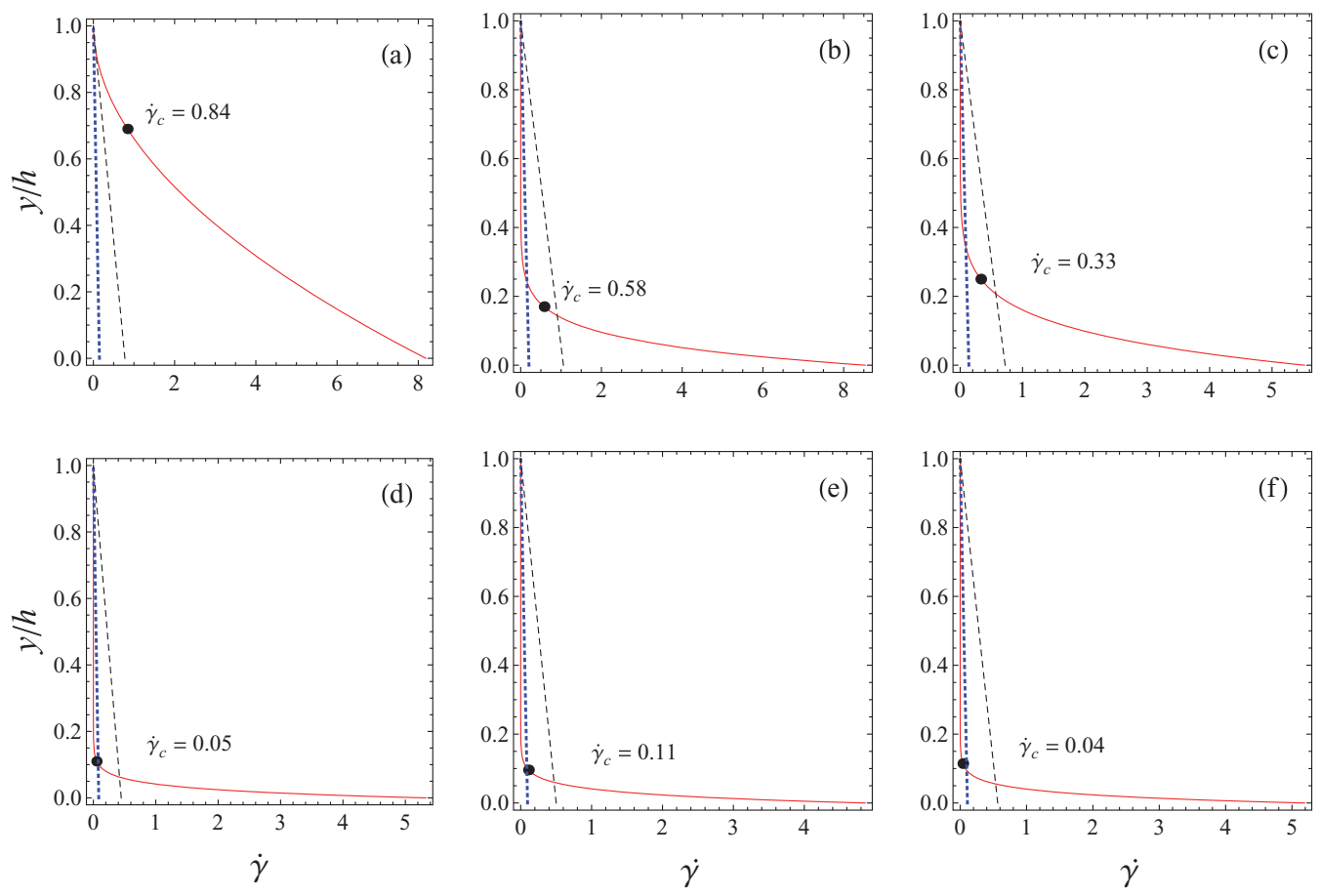

FIG. 12. Shear rate profiles for Run $\mathrm{H}(\bar{\phi}=0.595$, unsieved $)$. The solid line shows the shear rate $\dot{\gamma}$ obtained by differentiating the power-law function (3) fit on the experimental data. The dashed (black) line represents the theoretical shear rate $\dot{\gamma}=\Sigma_{x y} / \mu(\bar{\phi})$ with $\mu(\bar{\phi})$ the Krieger viscosity function (A3). The dotted (blue) line represents the shear rate $\dot{\gamma}=\Sigma_{x y} / \mu(\bar{\phi})$ with $\mu(\bar{\phi})$ the Zarraga viscosity function (A4).

\section{ACKNOWLEDGMENTS}

The work presented here was supported by the Swiss National Science Foundation under Grant No. 200021-105193/1 (a project called "Transient free-surface flows of concentrated suspensionsApplication to geophysical flows," funded by an R'Equip grant), the competence center in Mobile Information and Communication Systems (a center supported by the Swiss National Science Foundation under Grant No. 5005-67322, MICS project), the competence center in Environmental Sciences (TRAMM and APUNCH projects), and specific funds provided by EPFL (vice-présidence à la recherche). We are grateful to Belinda Bates for copyediting the paper. Gilbert Gruaz from EPFL/LMS carried out the oedometric tests and Bob de Graffenried from EPFL/LHE measured the particle size distribution.

\section{APPENDIX: THEORY}

\section{Scope and notation}

The goal of the Appendix is not to present a new theory and compare it with experimental data, but to see what we can learn about the rheology of granular suspensions from inclinedflume experiments and we can also see how predictions from existing theories compare with our experiments. Although an inclined flume is seldom categorized in the family of rheometers, it can provide interesting insights as the stress field is reasonably well-known. The flow depth profile and surface velocity as well as the front position over time are other sources of information, which have previously been used to investigate the rheological behavior of granular flows and concentrated suspensions.

In contrast with the procedure used this far in this paper, we will use physical variables instead of nondimensional variables. 


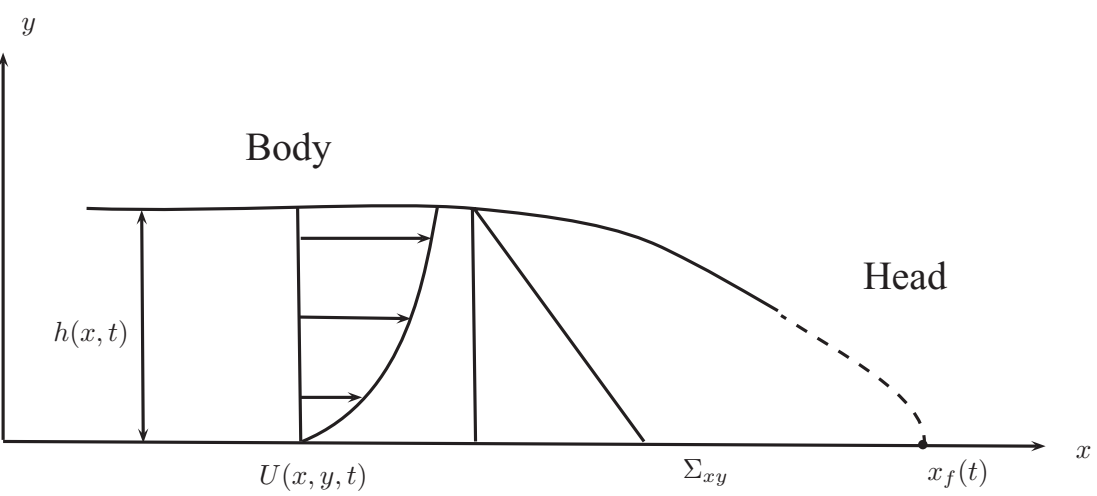

FIG. 13. Schematic defining the flow configuration.

Here we focus on slightly non-uniform time-dependent flows down a plane tilted at the angle $\theta$ to the horizontal (see Figure 13). The width is assumed to be infinitely large so that the sidewall effects are negligible. These flows are created by releasing a finite volume of material contained in a reservoir: initially a volume $A$ of suspension is contained in a reservoir and at time $t=0$, the material is released down a flume. The flow depth is denoted by $h(x, t)$. The front is the point at which the flow depth drops to zero. After the material has travelled a sufficiently long distance, it takes the form of a shallow elongated flow with a slow variation in the depth profile from front to tail. If, in addition, the flow is sufficient slow, then inertia can be neglected in the momentum balance equation. Under these assumptions, the momentum balance equations lead to a stress field of the form

$$
\begin{gathered}
\Sigma_{x y}=\rho g(h-y) \sin \theta, \\
\Sigma_{y y}=-\rho g(h-y) \cos \theta,
\end{gathered}
$$

where the normal stress is denoted by $\Sigma_{y y}$ and the shear stress by $\Sigma_{x y}$. In these equations, we have used a two-dimensional Cartesian coordinate system in which the $x$-axis points down the flume, the $y$-axis is in the direction of the upward-pointing normal, and the $z$-axis is in the cross-stream direction. The upper end of the reservoir is chosen to be the origin of the $x$-axis. The local velocity $u$ has components $u$ and $v$ in each of these directions; motion in the $z$-direction is not considered. Except where otherwise specified, $\dot{\gamma}=d u / d y$ is the shear rate.

In terms of dimensionless numbers, it can be easily shown that Eqs. (A1) and (A2) hold when the aspect ratio and Froude number satisfy $\epsilon \ll 1$ and $F r^{2} \epsilon \ll \sin \theta$. Note that Eqs. (A1) and (A2) hold true irrespective of the constitutive equation of the flowing material. These equations have been derived for quasi-steady uniform flows, i.e., $\partial_{x} h \ll 1$. This assumption is clearly violated within the tip region as the gradient of the free surface implies a significant increase in the shear stress. In the absence of inertia and normal-stress effects, it is possible to determine the effect of the free-surface gradient on the shear stress profile using lubrication theory. ${ }^{27}$ As we may meet the situation in which the normal stresses play a role, we will not go deeper into the analysis.

For this configuration, we study the spread of a finite volume of particle suspension down an inclined flume. The suspension is composed of non-colloidal particles within a Newtonian carrier fluid of viscosity $\mu_{f}$ and surface tension $\gamma$. Both solid and fluid phases have the same density $\rho_{f}=\rho_{p}=\rho$. The particle concentration is denoted by $\phi$ and its average throughout the bulk is called the (mean) solids fraction $\bar{\phi}$. The particles are assumed to be spheres of radius $a$.

We start with the simplest constitutive equation: we assume that the suspension is well-mixed and behaves like a homogeneous Newtonian fluid (see Subsection 2 of this Appendix). We then address the more realistic case of a heterogeneous suspension, in which the bulk viscosity $\mu$ is a function of the particle concentration $\phi$ (see Subsection 3 of this Appendix). 


\section{Macroviscous homogeneous fluid}

Let us assume that the bulk behaves like a homogeneous Newtonian fluid with viscosity $\mu$, which is given by an empirical equation such as the Krieger-Dougherty relation ${ }^{43}$

$$
\eta_{s}(\phi)=\frac{\mu(\phi)}{\mu_{f}}=\left(1-\frac{\phi}{\phi_{m}}\right)^{-\beta},
$$

with $\phi_{m}$ the maximum particle concentration and $\beta$ a constant exponent $\left(\beta=\frac{5}{2} \phi_{m}\right.$ or $\beta=2$ are common values). Other expressions have been proposed for the viscosity function, in particular, to better describe the viscosity behaviour in a wide range of flow conditions. ${ }^{34,44,45}$ For instance, Zarraga, Hill, and Leighton ${ }^{34}$ proposed a single-parameter viscosity function

$$
\eta_{s}(\phi)=\frac{\mu(\phi)}{\mu_{f}}=\exp (-2.34 \phi)\left(1-\frac{\phi}{\phi_{m}}\right)^{-3} .
$$

Integrating the constitutive equation $\Sigma_{x y}=\mu \dot{\gamma}$ and making use of the continuity equation leads to the velocity profiles:

$$
\begin{aligned}
& u(x, z, t)=\frac{1}{2} \frac{\rho g \sin \theta}{\mu} y(2 h-y), \\
& v(x, z, t)=\frac{1}{2} \frac{\rho g \sin \theta}{\mu} \frac{\partial h}{\partial x} y^{2} .
\end{aligned}
$$

A further integration leads to the depth-averaged velocity: $\bar{u}=\rho g h^{2} \sin \theta /(3 \mu)$. As the bulk mass balance equation is

$$
\frac{\partial h}{\partial t}+\frac{\partial h \bar{u}}{\partial x}=0
$$

we end up with a nonlinear advection equation for the evolution of $h$. This equation can be solved using the method of characteristics or by similarity solutions. It can be shown that the front position varies with time as ${ }^{28}$

$$
x_{f}=\left(\frac{9}{4} \frac{\rho \sin \theta A^{2}}{\mu} t\right)^{1 / 3},
$$

while the flow depth profile is given by

$$
h(x, t)=\sqrt{\frac{\mu}{\rho g \sin \theta}} \sqrt{\frac{x}{t}} .
$$

The equations do not hold in the close vicinity of the front as the flow depth drops to zero and the surface gradient is no longer negligible. As the thickness of the tip region is small relative to the body length, we will not go into more detail about the front behavior; see Refs. 27 and 28 for further information.

\section{Macroviscous inhomogeneous fluid}

Because of the shear in the cross-stream direction $y$, a concentration stratification occurs across the stream: particles migrate from the high- to low-shear regions, that is, from the basal layer to the free surface. This leads to a local change in the viscosity and thus velocity profile. Two additional equations are then needed to close the governing equations: the relationship between the bulk viscosity $\mu$ and particle concentration $\phi$, and the governing equation for $\phi(x, z, t)$. Simple empirical equations such as the Krieger-Dougherty relation (A3) are used to relate $\mu$ and $\phi$, while a more involved equation is needed to compute the $\phi(x, z, t)$ variations under the effect of a shear gradient. Several approaches have been proposed. ${ }^{11,12}$ Regardless of the underlying model of particle migration, the resulting governing equations are not tractable and must be solved numerically. Such numerical simulations allow us to infer some information that sheds light on the effect of particle migration in an "avalanching" suspension.

In simple-shear flows, particle migration leads to the gradual blunting of the velocity profile over time. Numerical solutions of particle migration models (paper under submission ${ }^{47}$ ) have shown 
that the deviation from the initial parabolic Newtonian profile can be described regarding the bulk as a power-law fluid with a time-dependent index $n$ and consistency $\kappa$, such that

$$
\Sigma_{x y}=\mu(\phi(y, t)) \dot{\gamma}=\kappa \dot{\gamma}^{n(t)} .
$$

Recall that, as stated in the body of this paper, this equation does not mean that suspensions are genuinely shear-thinning fluids, but that the constitutive equation $\Sigma_{x y}(\dot{\gamma}, \phi)$ can locally be approximated using a power-law model, whose index gives an idea of the importance of solids fraction inhomogeneities. It is also useful when there is no information about local $\phi$ variations.

Experiments and theory show that at long times, the index $n$ reaches an asymptotic value that depends solely on the mean solids fraction. The time to reach steady state is approximatively

$$
t_{s s} \sim \frac{H_{*}^{3}}{a^{2} U_{*}} .
$$

Our earlier experiments showed that for $t<t_{s s}, n$ decreased as $n \propto t^{-1 / 3}$ when the mean solids fraction was lower than $56 \%$, but more rapidly for $\bar{\phi}>0.56$. While theory predicts steady-state values of $n$ in the $0.2-0.7$ range for $\bar{\phi}$ in the 0.4-0.6 range, experiments have demonstrated that at the highest solids fraction, the steady-state value is significantly lower than the theoretical prediction and typically ranges from 0.01 to 0.1 .

As previously (Subsection 2 of this Appendix), we can derive the governing equation for the flow depth using the kinematic wave approximation

$$
\frac{\partial h}{\partial t}+\frac{\partial h \bar{u}}{\partial x}=0 \text { with } \bar{u}=\frac{n}{1+2 n}\left(\frac{\rho g \sin \theta}{\kappa}\right)^{1 / n} h^{1+1 / n},
$$

which leads to the following evolution equation for the front position

$$
x_{f}=\left(\frac{1+2 n}{1+n}\right)^{(1+n) /(1+2 n)}\left(\frac{\rho g \sin \theta}{\kappa} A^{n} t^{n}\right)^{1 /(1+2 n)},
$$

while the flow depth profile is given by

$$
h(x, t)=\sqrt[n+1]{\frac{\mu}{\rho g \sin \theta} \frac{x^{n}}{t^{n}}} .
$$

When $n$ is sufficiently close to unity (say, for $n>0.5$, which corresponds to solids fractions $\bar{\phi}<0.55$ ), there are few differences compared to the homogeneous case (Subsection 2 of this Appendix). Typically, for $n=1 / 2$, we get $x_{f} \propto t^{1 / 4}$ instead of $x_{f} \propto t^{1 / 3}$ for the homogeneous case. We expect that at the highest solids fractions $(\bar{\phi}>0.57)$, therefore $n$ value reaches 0.2 or lower, then $x_{f}(t)$ should provide an indication of the strength of particle migration.

${ }^{1}$ P. Coussot and C. Ancey, "Rheophysical classification of concentrated suspensions and granular pastes," Phys. Rev. E 59, 4445-4457 (1999).

${ }^{2}$ C. Ancey, P. Coussot, and P. Evesque, "A theoretical framework for very concentrated granular suspensions in a steady simple shear flow," J. Rheol. 43, 1673-1699 (1999).

${ }^{3}$ N. Mitarai and H. Nakanishi, "Granular flow: Dry and wet," Eur. Phys. J. Spec. Top. 204, 5-17 (2012).

${ }^{4}$ T. Divoux and J. C. Géminard, "Friction and dilatancy in immersed granular matter," Phys. Rev. Lett. 99, 258301 (2007).

${ }^{5}$ S. Deboeuf, G. Gauthier, J. C. Martin, Y. Yurkovetsky, and J. F. Morris, "Particle pressure in a sheared suspension: A bridge from osmosis to granular dilatancy," Phys. Rev. Lett. 102, 108301 (2009).

${ }^{6}$ D. M. Mueth, G. F. Debregeas, G. S. Karczmar, P. Eng, and S. R. Nagel, "Signatures of granular microstructure in dense shear flows," Nature (London) 406, 385-389 (2000).

${ }^{7}$ M. E. Cates, D. D. Haw, and C. B. Holmes, "Dilatancy, jamming, and the physics of granulation," J. Phys.: Condens. Matter 17, S2517-S2531 (2005).

${ }^{8}$ G. Ovarlez, Q. Barral, and P. Coussot, "Three-dimensional jamming and flows of soft glassy materials," Nature Mater. 9, 115-119 (2010).

${ }^{9}$ D. Prasad and H. K. Kytömaa, "Particle stress and viscous compaction during shear of dense suspensions," Int. J. Multiphase Flow 21, 775-785 (1995).

${ }^{10}$ C. Ancey and P. Coussot, "Transition from frictional to viscous regime for granular suspensions," C. R. Acad. Sci. Paris sér. IIb 327, 515-522 (1999).

${ }^{11}$ J. J. Stickel and R. L. Powell, "Fluid mechanics and rheology of dense suspensions," Annu. Rev. Fluid Mech. 37, 129-149 (2005). 
${ }^{12}$ J. F. Morris, "A review of microstructure in concentrated suspensions and its implications for rheology and bulk flow," Rheol. Acta 48, 909-923 (2009).

${ }^{13}$ C. Ancey, "Role of lubricated contacts in concentrated polydisperse suspensions," J. Rheol. 45, 1421-1439 (2001).

${ }^{14}$ B. Nsom, "The dam break problem for a hyperconcentrated suspension," Appl. Rheol. 10, 224-230 (2000).

${ }^{15}$ B. D. Timberlake and J. F. Morris, "Particle migration and free-surface topography in inclined plane flow of a suspension," J. Fluid Mech. 538, 309-341 (2005).

${ }^{16}$ C. Bonnoit, T. Darnige, E. Clément, and A. Lindner, "Inclined plane rheometry of a dense granular suspension," J. Rheol. 54, 65-79 (2010).

${ }^{17}$ T. Ward, C. Wey, R. Gidden, A. E. Hosoi, and A. L. Bertozzi, "Experimental study of gravitation effects in the flow of a particle-laden thin film on an inclined plane," Phys. Fluids 21, 083305 (2009).

${ }^{18}$ J. Zhou, B. Dupuy, A. L. Bertozzi, and A. E. Hosoi, "Theory for shock dynamics in particle-laden thin films," Phys. Rev. Lett. 94, 117803 (2005).

${ }^{19} \mathrm{C}$. Ancey, "Solving the Couette inverse problem by using a wavelet-vaguelette decomposition," J. Rheol. 49, 441-460 (2005).

${ }^{20}$ G. Ovarlez, F. Bertrand, and S. Rodts, "Local determination of the constitutive law of a dense suspension of noncolloidal particles through magnetic resonance imaging," J. Rheol. 50, 259-292 (2006).

${ }^{21}$ A. Fall, F. Bertrand, G. Ovarlez, and D. Bonn, "Yield stress and shear banding in granular suspensions," Phys. Rev. Lett. 103, 178301 (2009).

${ }^{22}$ S. Wiederseiner, N. Andreini, G. Épely-Chauvin, and C. Ancey, "Refractive index matching in concentrated particle suspensions: A review," Exp. Fluids 50, 1183-1206 (2011).

${ }^{23} \mathrm{~N}$. Andreini, "Dam break of Newtonian fluids and granular suspensions: Internal dynamics measurements," Ph.D. dissertation (Ecole Polytechnique Fédérale de Lausanne, 2012).

${ }^{24}$ F. K. Hansen and G. Rødsrud, "Surface tension by pendant drop: I. A fast standard instrument using computer image analysis," J. Colloid Interface Sci. 141, 1-9 (1991).

${ }^{25}$ M. Raffel, C. E. Willert, S. T. Wereley, and J. Kompenhans, Particle Image Velocimetry (Springer, Berlin, 2007).

${ }^{26}$ J. K. Sveen, "An introduction to MatPIV," Mechanics and Applied Mathematics, Technical Report No. 2 (Department of Mathematics, University of Oslo, 2004).

${ }^{27}$ N. Andreini, G. Epely-Chauvin, and C. Ancey, "Internal dynamics of Newtonian and viscoplastic fluid avalanches down a sloping bed," Phys. Fluids 24, 053101 (2012).

${ }^{28}$ C. Ancey, S. Cochard, and N. Andreini, "The dam-break problem for viscous fluids in the high-capillary-number limit," J. Fluid Mech. 624, 1-22 (2009).

${ }^{29}$ G. Y. Onoda and E. Liniger, "Random loose packings of uniform spheres and the dilatancy onset," Phys. Rev. Lett. 64, 2727-2730 (1990)

${ }^{30}$ A. P. Shapiro and R. F. Probstein, "Random packings of spheres and fluidity limits of monodisperse and bidisperse suspensions," Phys. Rev. Lett. 68, 1422-1425 (1992).

${ }^{31}$ M. Jerkins, M. Schröter, H. L. Swinney, T. J. Senden, M. Saadatfar, and T. Aste, "Onset of mechanical stability in random packings of frictional spheres," Phys. Rev. Lett. 101, 018301 (2008).

${ }^{32}$ P. R. Nott and J. F. Brady, "Pressure-driven flow of suspensions: Simulation and theory," J. Fluid Mech. 275, 157-199 (1994).

${ }^{33}$ H. A. Barnes, "A review of the slip (wall depletion) of polymer solutions, emulsions, and particle suspensions in viscometers: Its cause, character, and cure," J. Non-Newtonian Fluid Mech. 56, 221-251 (1995).

${ }^{34}$ I. E. Zarraga, D. A. Hill, and D. T. Leighton, "The characterization of the total stress of concentrated suspensions of noncolloidal spheres in Newtonian fluids," J. Rheol. 44, 185-221 (2000).

${ }^{35}$ J. M. N. T. Gray and C. Ancey, "Particle size-segregation, recirculation, and deposition at coarse particle rich flow fronts," J. Fluid Mech. 629, 387-423 (2009).

${ }^{36}$ R. I. Tanner, Engineering Rheology, Oxford Engineering Science Series (Clarendon, Oxford, 1988).

${ }^{37}$ E. Couturier, F. Boyer, O. Pouliquen, and E. Guazzelli, "Suspensions in a tilted trough: Second normal stress difference," J. Fluid Mech. 686, 26-39 (2011).

${ }^{38}$ E. Brown and H. M. Jaeger, "Dynamic jamming point for shear thickening suspensions," Phys. Rev. Lett. 103, 086001 (2009).

${ }^{39}$ C. Bonnoit, J. Lanuza, A. Lindner, and E. Clément, "Mesoscopic length scale controls the rheology of dense suspensions," Phys. Rev. Lett. 105, 108302 (2010)

${ }^{40}$ E. Brown and H. M. Jaeger, "The role of dilation and confining stresses in shear thickening of dense suspensions," J. Rheol. 56, 875-923 (2012).

${ }^{41}$ P. Snabre and B. Pouligny, "Size segregation in a fluid-like or gel-like suspension settling under gravity or in a centrifuge," Langmuir 24, 13338-13347 (2008).

${ }^{42}$ N. Huang, G. Ovarlez, F. Bertrand, S. Rodts, P. Coussot, and D. Bonn, "Flow of wet granular materials," Phys. Rev. Lett. 94, 028301 (2005)

${ }^{43}$ I. M. Krieger and T. J. Dougherty, "A mechanism for non-Newtonian flow in suspensions of rigid spheres," Trans. Soc. Rheol. 3, 137-152 (1959).

${ }^{44}$ J. F. Morris and F. Boulay, "Curvilinear flows of noncolloidal suspensions: The role of normal stresses," J. Rheol. 43, 1213-1238 (1999).

${ }^{45}$ F. Boyer, E. Guazzelli, and O. Pouliquen, "Unifying suspension and granular rheology,” Phys. Rev. Lett. 107, 188301 (2011).

${ }^{46}$ N. Andreini, C. Ancey, and G. Epely-Chauvin, "Granular suspension avalanches. II. Plastic regime,” Phys. Fluids 25, $033302(2013)$

${ }^{47}$ C. Ancey, N. Andreini, and G. Epely-Chauvin, "The dam-break problem for concentrated suspensions of neutrally buoyant particles," J. Fluid Mechanics (to be published). 